ARTICLE

DOI: $10.1038 / \mathrm{s} 41467-017-00632-2$

\title{
P-body proteins regulate transcriptional rewiring to promote DNA replication stress resistance
}

\author{
Raphael Loll-Krippleber ${ }^{1} \&$ Grant W. Brown (1) ${ }^{1}$
}

mRNA-processing (P-) bodies are cytoplasmic granules that form in eukaryotic cells in response to numerous stresses to serve as sites of degradation and storage of mRNAs. Functional P-bodies are critical for the DNA replication stress response in yeast, yet the repertoire of $\mathrm{P}$-body targets and the mechanisms by which $\mathrm{P}$-bodies promote replication stress resistance are unknown. In this study we identify the complete complement of mRNA targets of $\mathrm{P}$-bodies during replication stress induced by hydroxyurea treatment. The key P-body protein Lsm1 controls the abundance of HHT1, ACF4, ARL3, TMA16, RRS1 and YOX1 mRNAs to prevent their toxic accumulation during replication stress. Accumulation of YOX1 mRNA causes aberrant downregulation of a network of genes critical for DNA replication stress resistance and leads to toxic acetaldehyde accumulation. Our data reveal the scope and the targets of regulation by P-body proteins during the DNA replication stress response.

\footnotetext{
${ }^{1}$ Department of Biochemistry and Donnelly Centre, University of Toronto, 160 College Street, Toronto, ON, Canada M5S 3E1. Correspondence and requests for materials should be addressed to G.W.B. (email: grant.brown@utoronto.ca)
} 
$\mathrm{D}$ NA replication is influenced by both environmental and internal cues. Chemical agents or metabolic by-products can cause DNA modifications that stall or slow DNA replication forks ${ }^{1}$. Similarly, DNA secondary structures or the presence of the transcription machinery on the DNA can act as barriers to replication forks ${ }^{1}$. In order to fully and accurately replicate the genome in presence of these perturbants, eukaryotic cells encode a multi-faceted response referred to as the replication stress response ${ }^{1}$. Excess replication stress causes mutations, genome rearrangements, and loss of genetic material, and can result in cell death and disease ${ }^{1,2}$. Therefore, DNA replication as well as the pathways controlling the response to stresses affecting this critical biological process must be tightly regulated.

In the model eukaryote Saccharomyces cerevisiae, the DNA replication stress response has been extensively studied at both the single-gene/protein level and at the genome/proteome scale $^{3-10}$. DNA replication stress induces extensive changes in intracellular protein localization, with as much as $6 \%$ of the proteome re-localizing upon drug-induced DNA replication stress $8,9,11,12$. Although much of the global protein location repatterning that occurs during replication stress involves nuclear and intra-nuclear protein localizations, one of the most prominent type of localization change is the coalescence of proteins to form foci in the cytoplasm ${ }^{8,9}$. Formation of cytoplasmic foci is a characteristic of mRNA-processing bodies (P-bodies) and indeed, the group of proteins that re-localize to cytoplasmic foci during replication stress is highly enriched in P-body components ${ }^{8}$.

P-bodies are cytoplasmic membrane-free granules that contain non-translating mRNAs and proteins involved in mRNA decapping and degradation ${ }^{13}$. Critical P-body components include the decapping enzyme Dcp2, the decapping activator Dcp1, the decapping enhancers Edc1-3, Dhh1, Pat1 and Lsm1-7, and the $5 '-3^{\prime}$ exonuclease Xrn1, which together determine the decapping or degradation rate of mRNAs ${ }^{13}$. P-bodies are present in nonstressed cells, however their number and size increase in response to diverse conditions including glucose starvation, osmotic stress, growth to stationary phase, and DNA replication stress $8,14$. P-body formation during replication stress has unique genetic requirements ${ }^{8}$, suggesting that P-body formation, and perhaps the regulation of mRNAs that are P-body targets, is a specifically controlled stress response. Furthermore, deletion of P-body genes confers sensitivity to DNA replication stress ${ }^{8}$, and causes genome instability ${ }^{15}$. As available data indicate that $\mathrm{P}$-body regulation of mRNA targets is relevant to DNA replication stress resistance, we sought to identify the network of P-body targets to understand how they contribute to cellular fitness during DNA replication stress.

Here, we define the network of mRNAs regulated by P-body proteins during DNA replication stress. We leverage the transcriptome data in combination with a systematic genetic suppression screen to identify the P-body targets that are important for DNA replication stress resistance. Our genomic analyses converge on the transcription factor Yox1, whose accumulation is toxic in cells experiencing replication stress, and whose mRNA localizes to P-bodies. Yox1 toxicity is likely due to its transcriptional repression function, as we found that Yoxl downregulates genes that are critical for resistance to replication stress. In particular, we show that $A L D 6$ de-repression is critical to prevent the toxic accumulation of acetaldehyde. Thus, we identify a key DNA replication stress resistance pathway regulated by the P-body target YOX1.

\section{Results}

Deletion of LSMI extensively remodels the transcriptome. LSM1 is essential for the regulation of functional P-body formation, and for the degradation or stability of specific mRNA targets $^{16-18}$. In order to identify the complement of mRNA targets of P-body regulation, we used RNA-seq to profile the transcriptomes of wild-type (WT) and lsm $1 \Delta$ cells, in the presence and absence of the replication stress inducing drug hydroxyurea (HU) (Fig. 1a). The experiment was performed in duplicate on total RNA depleted of rRNA, and differential expression was assessed using the Tuxedo protocol ${ }^{19}$. Genes showing differential expression with a statistical $P$-value less than 0.05 after Benjamini-Hochberg correction ${ }^{19}$ were considered as hits in our analysis. The transcriptome was greatly affected upon deletion of LSM1 (Fig. 1b, c; Supplementary Data 1). As expected, given the role of Lsm1 in RNA degradation ${ }^{16-18}$, we found that 333 mRNAs increased in abundance when LSM1 was deleted. Unexpectedly, a similar number of mRNAs (258) decreased in abundance (Fig. 1b, c; Supplementary Data 1). Although we have not determined whether these mRNAs are direct P-body targets, it is possible that association of mRNAs with Lsm1 is in some cases protective and prevents exosome-dependent degradation ${ }^{20}$ Alternatively, absence of LSM1 could stabilize transcriptional repressors, resulting indirectly in mRNA abundance decreases, as has been observed in cells lacking the $5^{\prime}-3^{\prime}$ RNA exonuclease $\mathrm{Xrn} 1^{21}$.

In WT cells, HU treatment induced considerable change in mRNA abundance. Hundreds of transcripts showed increased or decreased abundance after only $1 \mathrm{~h}$ of HU treatment (Supplementary Fig. 1 and Supplementary Data 1). After $4 \mathrm{~h}$ of HU treatment 1134 and 1178 transcripts showed increased or decreased abundance, respectively (Supplementary Fig. 1). We found reasonable positive correlations between our data and previous microarray transcriptomic studies in $\mathrm{HU}^{6}$ or methyl methanesulfonate (MMS) ${ }^{7}, 22$ (Supplementary Fig. 2a, b). The correlation with data obtained using a distinct replication stress agent, MMS, indicates that a substantial fraction of the transcriptional program that we identified is due to DNA replication stress (Supplementary Fig. 2b). Some changes in gene expression that we observe are likely the result of cell-cycle synchronization in early $S$ phase that occurs upon HU addition. Both WT and $l s m 1 \Delta$ undergo similar HU arrests, however, and so cell-cycle effects are unlikely to impinge on the identification of differentially expressed genes when WT and $l s m 1 \Delta$ are compared (Supplementary Fig. 3).

Deletion of LSM1 had a dramatic effect on mRNA abundance during HU treatment. Between 499 (1 h after HU treatment) and 1203 transcripts ( $4 \mathrm{~h}$ after HU treatment) increased in abundance in the $l \operatorname{sm} 1 \Delta$ strain (Fig. 1b, c and Supplementary Data 1) compared to the WT control. The unexpected transcript abundance decreases that we observed in the absence of $\mathrm{HU}$ treatment in $1 \mathrm{sm} 1 \Delta$ were more extensive in the presence of $\mathrm{HU}$, where 322 to $1051 \mathrm{mRNAs}$ decreased in abundance. Almost half of the differentially expressed genes in $l s m 1 \Delta$ cells were downregulated, again indicating an unanticipated positive or protective role for P-body proteins in the regulation of gene expression (Fig. 1b, c and Supplementary Data 1). Overall, however, the distribution of the mRNA abundance ratios for the differentially expressed genes showed a statistically supported positive bias, indicating that the stabilizing effect on RNAs observed upon deletion of LSM1 is stronger than the unexpected destabilizing effect (Fig. 1d). Interestingly, a large fraction (as much as 53\%) of the transcripts whose abundance was affected by HU treatment and deletion of LSM1 were also affected by MMS treatment, suggesting that Lsm1-regulated transcripts change in abundance during DNA replication stress in general (Supplementary Fig. 2c).

Finally, to confirm that the differentially expressed genes that we identified were independent of the data analysis 
method used, we applied two additional analyses to initial analysis were also identified using EBSeq or identify differentially expressed genes: $\mathrm{EBSeq}^{23}$ and edgeR ${ }^{24}$. edgeR, depending on the time point analyzed (Supplementary Between 34 and $79 \%$ of the genes identified in our Table 1).

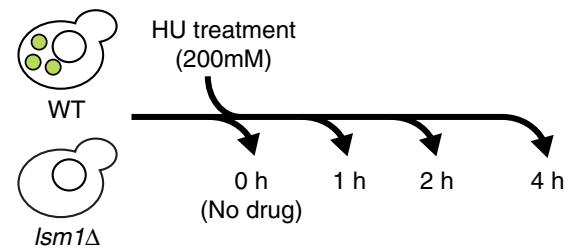

d

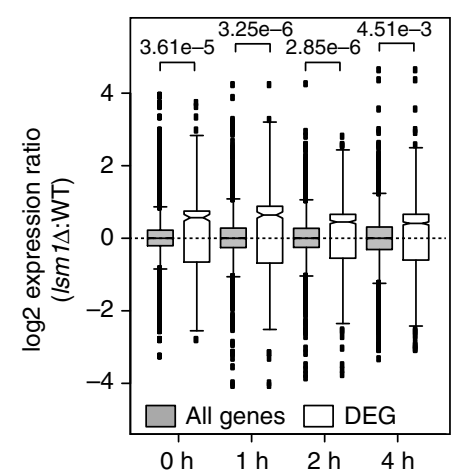

f

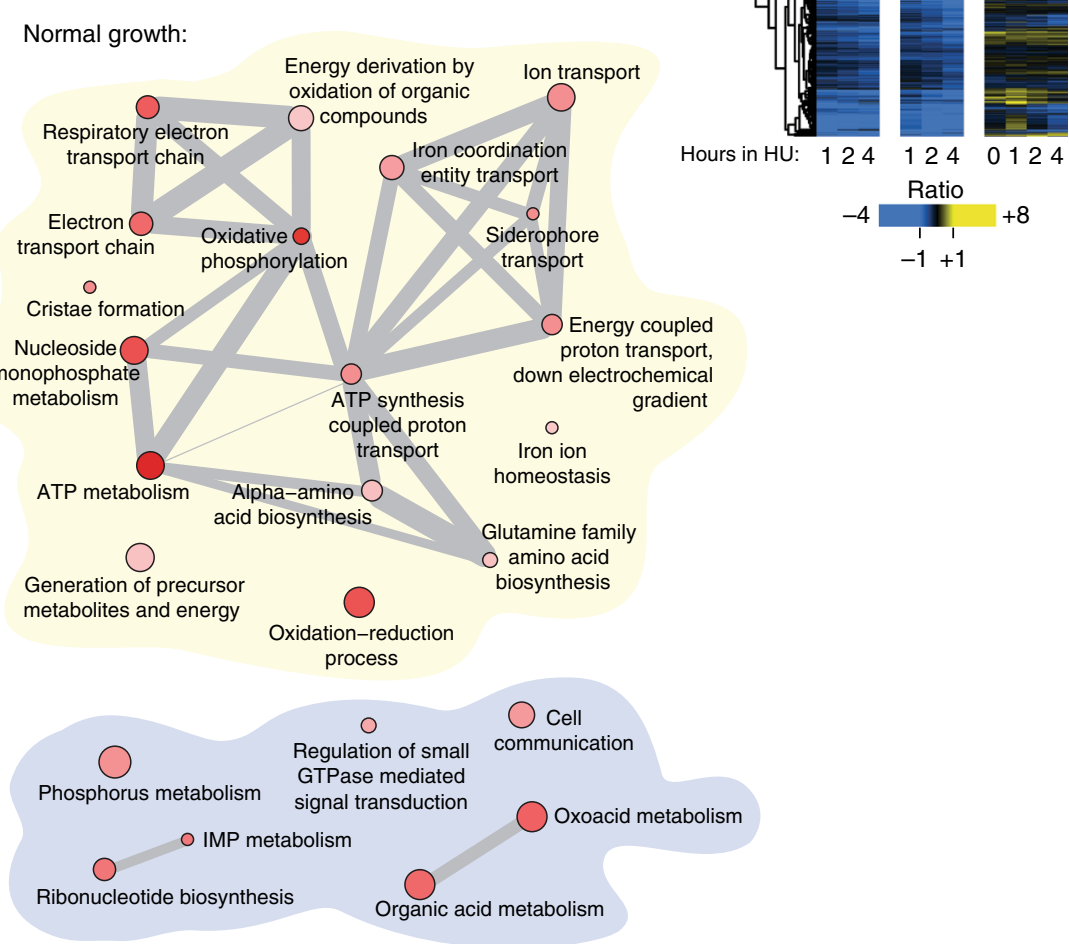

b

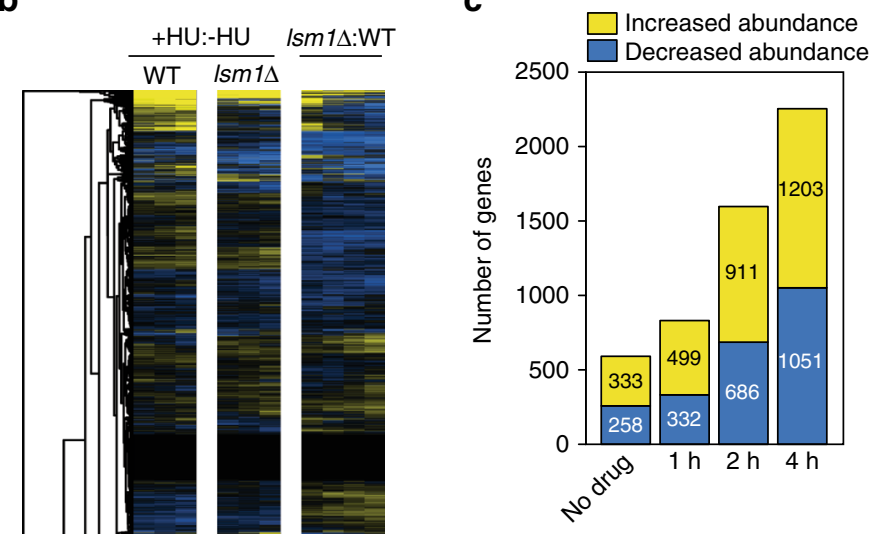

e

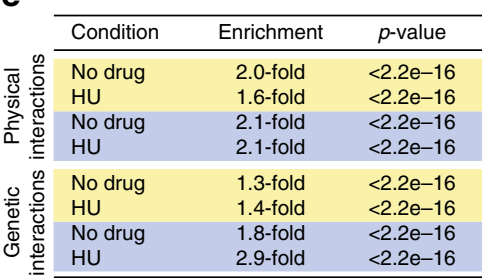

g
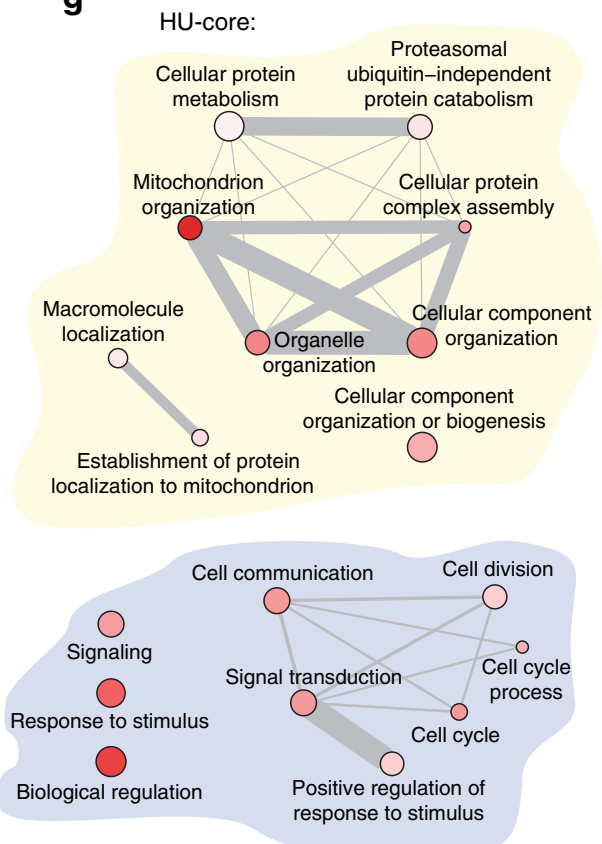

Fig. 1 The transcriptome is extensively remodeled when functional P-bodies are absent. a Overview of the RNA-seq strategy used to measure RNA abundance in $1 s m 1 \Delta$ cells. b Gene expression log2 ratios plotted as a heatmap. Expression ratios comparing HU treatment to the untreated condition (+HU: $-\mathrm{HU}$ ), in wild type (WT) and Ism1 $1 \Delta$, are plotted on the left. Expression ratios comparing Ism1 $1 \Delta$ cells to WT cells during normal growth and exposure to HU $(I s m 1 \Delta: \mathrm{WT})$ are plotted on the right. $\mathbf{c}$ The number of differentially expressed transcripts in $1 \mathrm{sm} 1 \Delta$ during replication stress is plotted. $\mathbf{d}$ The distributions of the Ism1 1 :WT expression ratios are displayed as boxplots for all genes (gray) and the differentially expressed genes (white). P-values are from unpaired Wilcoxon tests. e Summary of interaction enrichments for mRNAs with increased abundance in $I s m 1 \Delta$ (yellow) and with decreased abundance in $I s m 1 \Delta$ (blue) during normal growth (no drug) and during replication stress (HU). P-values were calculated using a binomial test. f GO term enrichment networks for mRNAs that were differentially expressed in $1 s m 1 \Delta$ during normal growth, for mRNAs with increased abundance (yellow) and mRNAs with decreased abundance (blue). Nodes are shaded according to $P$-values (darkest red indicating the smallest $P$-value). Node size indicates the frequency at which a given $\mathrm{GO}$ term is found in the S. cerevisiae genome. Edges connect highly similar GO terms and edge thickness represents degree of similarity. $\mathbf{g}$ GO term enrichment networks for mRNAs that were differentially expressed in $1 \mathrm{sm} 1 \Delta$ during $\mathrm{HU}$ treatment (HU-core genes) 
a

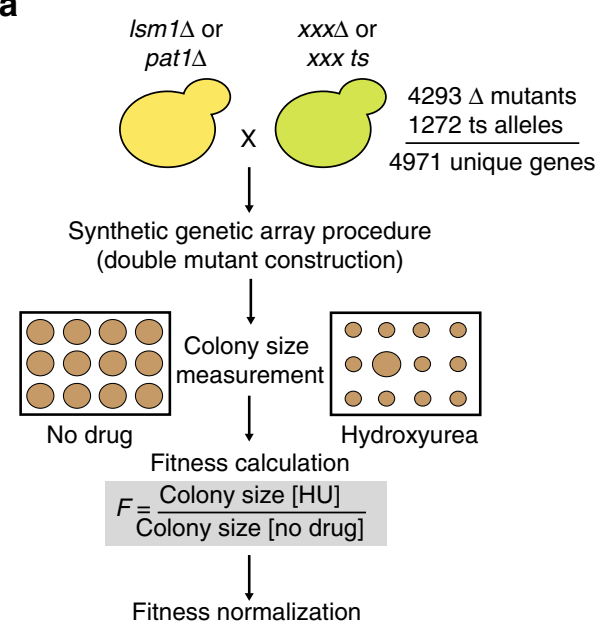

(Z-score determination for each plate)

Candidate suppressor genes

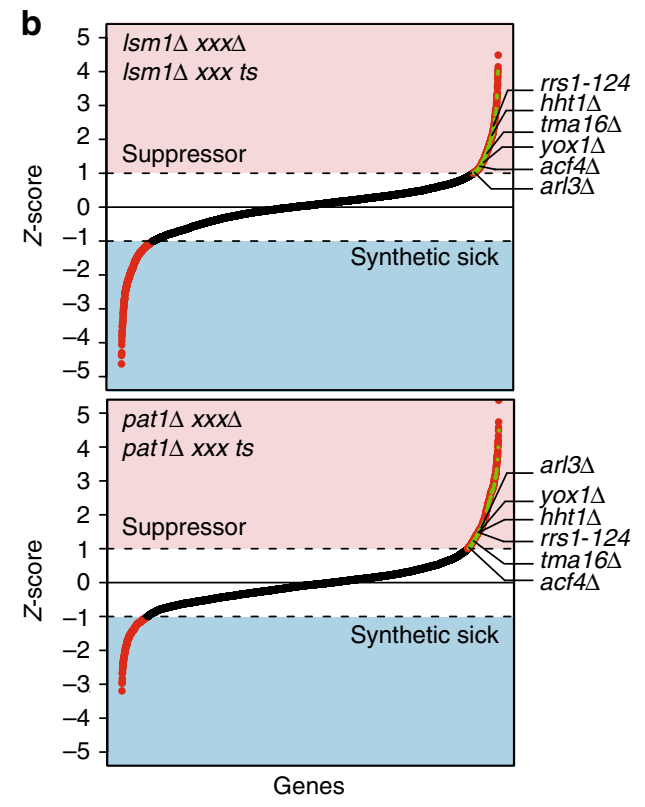

C

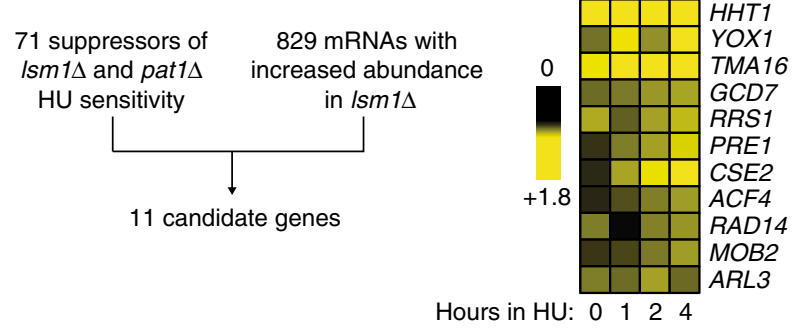

d

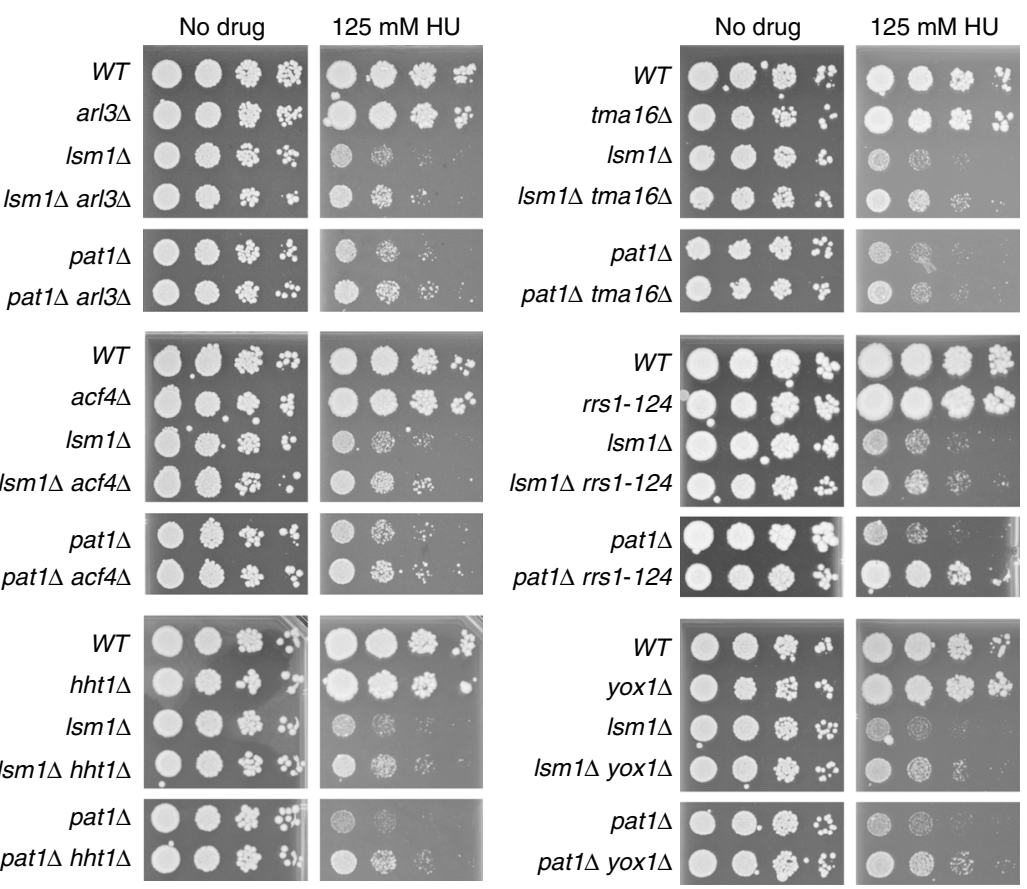

Fig. 2 Combining a systematic suppressor screen with mRNA abundance data to identify P-body targets in replication stress. a Overview of the screen to identify suppressors of the $\mathrm{HU}$ sensitivity of P-body mutants. b Z-score normalized HU fitness values for the Ism1 $x x x$ and pat1 $x x x$ double mutants. Genes that were identified in both screens are indicated in green. Validated hits are indicated on the right. $\mathbf{c}$ The mRNA abundance during replication stress is displayed as a heatmap for eleven candidate genes from the intersection of the suppressor screen and mRNA abundance data. The log2 Ism1 $\Delta:$ WT ratio is plotted for the indicated time points. $\mathbf{d} \mathrm{HU}$ sensitivity assay for the 6 validated candidate genes among the 11 genes identified in c. Ten-fold dilutions of an overnight culture were spotted on YPD medium with or without HU. e Biological functions of the validated candidate genes

Differentially expressed genes are functionally enriched. Mining existing databases ${ }^{25}$ revealed modest enrichments for both genetic and physical interactions in differentially expressed genes in $l s m 1 \Delta$, both during normal growth and in DNA replication stress (Fig. 1e). These data indicate that genes that are differentially expressed in $l s m 1 \Delta$ relative to WT are more likely to share functional biological connections, and this is true both during unperturbed growth and in the presence of replication stress.

We further assessed functional connections among the differentially expressed genes by testing for enrichment of gene ontology (GO) biological process terms. Among the genes with increased mRNA abundance in $l s m 1 \Delta$ we noted enrichment for
GO terms related to ATP synthesis, particularly genes encoding components of the electron transport chain, suggesting a role for P-bodies in regulating energy transactions during normal cellular growth (Fig. 1f and Supplementary Data 2). Beside ribonucleotide metabolism, little additional functional enrichment was observed among the genes with decreased expression in $l s m 1 \Delta$, suggesting that mRNA abundance decreases in the absence of functional P-bodies during normal cell growth could be largely non-specific.

To assess functional enrichment in the presence of replication stress, we defined a core set of HU-responsive genes as the genes being differentially expressed only upon HU exposure and at two consecutive time points (601 increased abundance and 417 decreased abundance). In addition, we removed genes known to 
a

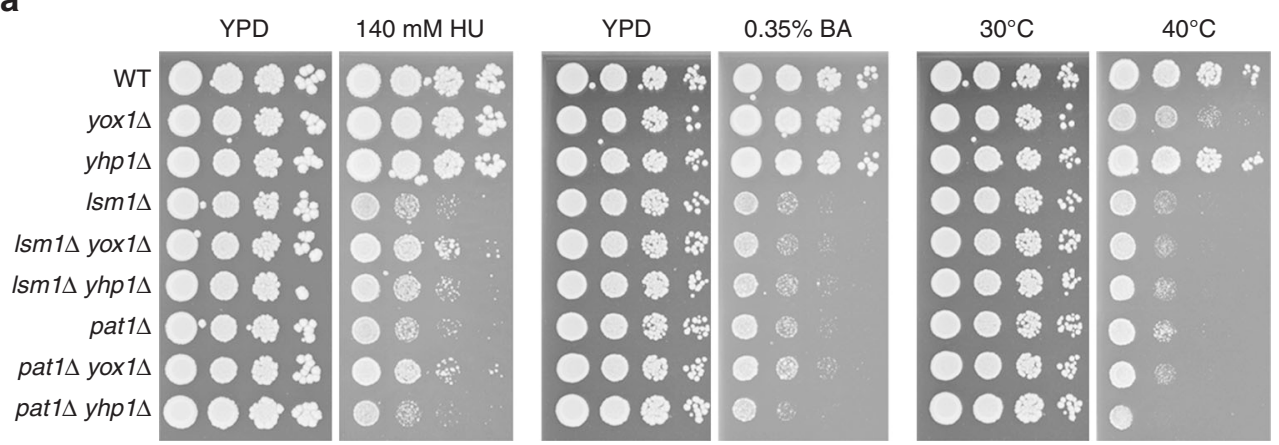

b

\begin{tabular}{|c|c|c|c|c|}
\hline & \multicolumn{2}{|c|}{$2 \%$ raffinose } & \multicolumn{2}{|c|}{$0.01 \%$ galactose $+2 \%$ raffinose } \\
\hline & SC-ura & $45 \mathrm{mM} \mathrm{HU}$ & SC-ura & $45 \mathrm{mM} \mathrm{HU}$ \\
\hline$W T+$ empty vector & $00 \Leftrightarrow 8$ & 00025 & $x-1=$ & $00<3$ \\
\hline$W T+G A L 1-10 p r-Y O X 1$ & 000 & $0000 \leqslant$ & 5 & 0002 का \\
\hline Ism1 $1 \Delta+$ empty vector & $0<\infty$ & 0 (2) & $00 \cdots$ & $00 \%$ \\
\hline Ism1 $1 \Delta+$ GAL1-10pr-YOX1 & $000=3$ & 00 雷 & 004 & 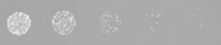 \\
\hline pat1 $1 \Delta+$ empty vector & 000 a & $00 \% \approx$ & $0.0 \Leftrightarrow$ & 00 然 \\
\hline pat1 + GAL1-10pr-YOX1 & $000<4$ & $00 \%$ & ○. & 0 \\
\hline
\end{tabular}

Fig. 3 YOX1 toxicity is specific to DNA replication stress. a Serial 10-fold dilutions of the indicated strains were spotted on YPD with or without HU, boric acid $(B A)$ or at high temperature $\left(40^{\circ} \mathrm{C}\right)$. Plates were imaged after 2-3 days of growth to assess the sensitivity of each strain to each treatment. b Serial 5-fold dilutions of WT (BY4741), Ism1 or pat1A cells containing pBY011 (empty vector) or pBY011-YOX1 (GAL1-10pr-YOX1) plasmids were spotted on synthetic complete medium lacking uracil and containing raffinose or raffinose and galactose, with or without HU. Plates were imaged after 7 days of growth to assess the effect of YOX1 expression on $\mathrm{HU}$ sensitivity

be involved in the general environmental stress response from our core set of HU-responsive genes (72 genes in the increased abundance set (42 upregulated in the environmental stress response) and 49 genes in the decreased abundance set (35 downregulated in the environmental stress response) $)^{26}$. Genes engaged in several aspects of mitochondrial function, particularly protein import into the mitochondria, were enriched in the set of genes whose mRNA abundance increased in HU in $l s m 1 \Delta$, as were genes encoding proteasome subunits (Fig. 1g and Supplementary Data 2). Genes encoding cell-cycle regulators were enriched in the genes whose mRNA abundance decreased in HU in $l s m 1 \Delta$ cells (Fig. $1 \mathrm{~g}$ and Supplementary Data 2). The functional enrichments suggest that P-bodies downregulate mitochondrial biogenesis and protein degradation and upregulate genes involved in controlling cell-cycle progression during HU-induced replication stress.

Thus, in addition to the major regulatory effect that functional P-bodies exert on gene expression in unperturbed cells, we define an additional layer of regulation of transcript abundance by P-body proteins, both positive and negative, that is evident when replication stress is present. When compared to unperturbed cells, P-body proteins regulate different cellular processes during exposure to replication stress, consistent with a specific biological role for P-bodies in the DNA replication stress response.

Suppressors of the HU sensitivity of P-body mutants. Having established that the P-body protein Lsm1, either directly or indirectly, regulates the abundance of mRNAs during replication stress, we sought to identify mRNAs whose downregulation at $\mathrm{P}$-bodies is important for replication stress resistance. We reasoned that the HU sensitivity of P-body mutants is due to the increased abundance of critical P-body target mRNAs. Therefore, deletion of the genes encoding these targets should suppress the
HU sensitivity of P-body mutants (Fig. 2a). Since LSM1 and PAT1 share the most genetic interactions of any two P-body components $^{27}$, we conducted suppression screens of both $l s m 1 \Delta$ and pat $1 \Delta$ (Fig. $2 \mathrm{~b}$ and Supplementary Data 3), using the Synthetic Genetic Array (SGA) method ${ }^{28}$ to systematically inactivate 4971 genes $(\sim 76 \%$ of the genome) in an $l s m 1 \Delta$ or pat $1 \Delta$ background. We assessed the fitness of every double mutant, by measuring and comparing colony size in presence and absence of $\mathrm{HU}$, in triplicate. Fitness values were normalized in order to remove plate and/or pinning artefacts, and converted to $Z$-scores. The $Z$-score distribution was similar in both $l \operatorname{sm} 1 \Delta$ and pat $1 \Delta$ strain backgrounds (Fig. 2b). Double mutants with a $Z$-score greater than +1 were considered to be HU sensitivity suppressors. Because the individual suppressor screens had a low true positive rate $(27 \% ; 3$ candidates validated, after strain re-construction, out of 11 randomly picked suppressor hits) we limited our analysis to suppressors identified in both the $l \operatorname{sm} 1 \Delta$ and pat $1 \Delta$ screens. A total of 71 genes, when inactivated, were able to suppress both $l s m 1 \Delta$ and pat1 $\Delta$ HU sensitivity. Among these candidate P-body target genes, 11 also have increased transcript abundance in the absence of LSM1 at 2 or more consecutive times after HU treatment (Fig. 2c). Independent re-construction of the pat $1 \Delta$ and $l s m 1 \Delta$ double mutants with each of the 11 genes resulted in validation of 6 putative target genes: ARL3, ACF4, HHT1, TMA1, RRS1 and YOX1 and increased mRNA abundance in $\mathrm{HU}$ for 5 of these transcripts was confirmed by two independent data analysis methods. ACF4 was confirmed by edgeR but not by EBSeq (Supplementary Table 2). Mutations in each suppressed both $l s m 1 \Delta$ and pat $1 \Delta \mathrm{HU}$ sensitivity (Fig. $2 \mathrm{~d}$ ). In no case was suppression complete, suggesting that multiple P-body targets contribute to fitness during the replication stress response. The biological function of the 6 genes is described in Fig. 2e. Two genes (RRS1 and TMA16) encode ribosome-associated proteins ${ }^{29}, 30$, suggesting that downregulation of protein translation capacity is important for DNA replication stress resistance. 
Two (ACF4 and YOX1) encode phosphorylation targets of the cyclin dependant kinase Cdc28 $8^{31}$. Importantly, among the six genes we identified, one (HHT1) is known to be a P-body target during replication stress ${ }^{32}$. Therefore, our strategy identified five new genes whose expression is toxic during replication stress when P-body function is compromised.

YOX1 regulation by $\mathrm{P}$-bodies is critical during $\mathrm{HU}$ stress. Yox1 is a homeobox transcriptional repressor that regulates the expression of cell-cycle regulated and DNA replication genes ${ }^{33}$. Together with Mcm1, Yox1 binds to the promoters of genes whose regulation is critical during the M/G1 transition of the cell cycle ${ }^{33}$. Inactivation of the Yoxl protein is important during replication stress in Schizosaccharomyces pombe ${ }^{34}$, and overexpression of YOX1 causes a dramatic increase in chromosome loss in S. cerevisiae ${ }^{35}$. DNA replication stress causes Yox1 to re-localize from the nucleus to the cytoplasm ${ }^{8}$, suggesting that expression of Yox1 targets could play an important role in the replication stress response. Given its connections to the replication stress response, we asked if YOX1 expression is regulated by P-body proteins.

We first confirmed that YOX1 mRNA levels increase in P-body mutants. When we examined YOX1 mRNA by quantitative reverse transcription PCR (RT-PCR), we found that in both $l s m 1 \Delta$ and pat1 $Y O X 1$ mRNA accumulated relative to the WT cells (Supplementary Fig. 4). Deletion of PAT1 abolishes P-body formation during replication stress ${ }^{8}$, while loss of LSM1 a

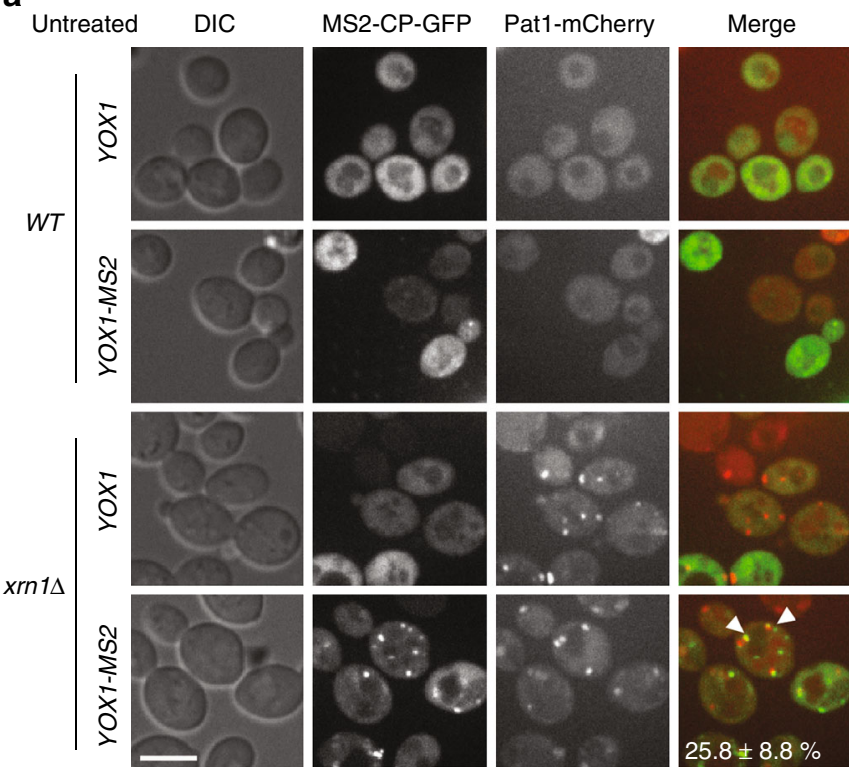

$200 \mathrm{mM} \mathrm{HU}$

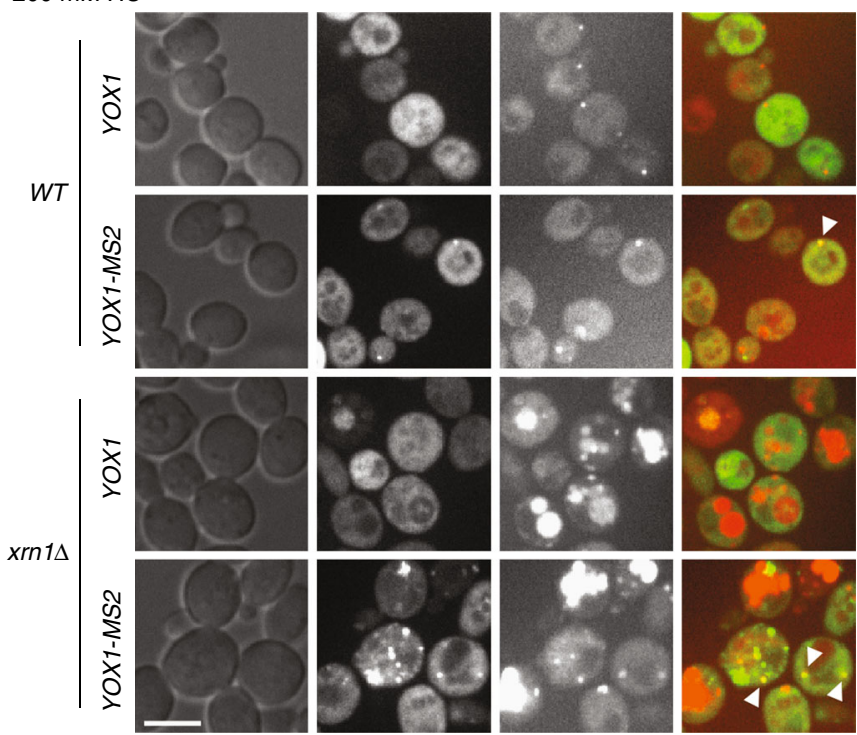

b

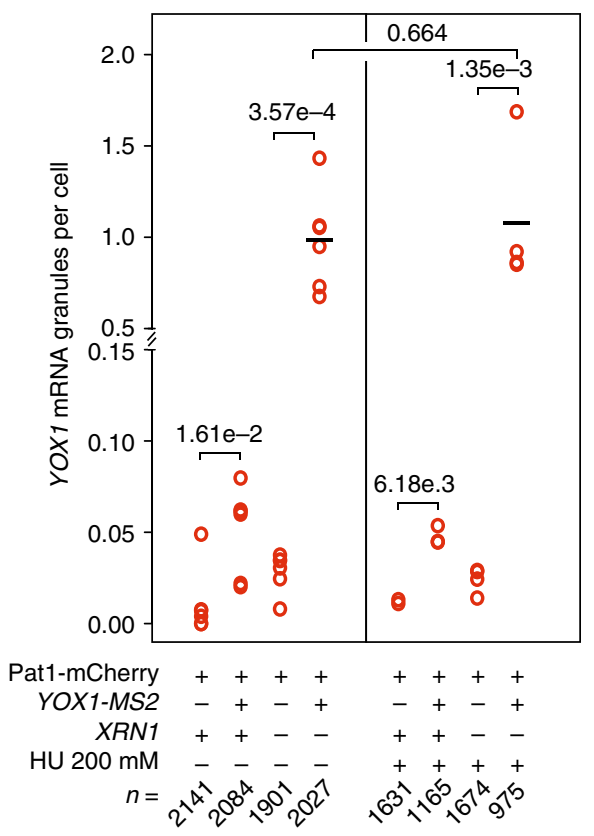

C

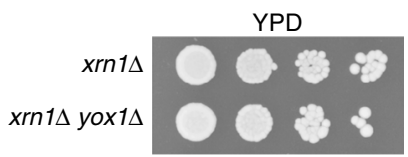

$140 \mathrm{mM} \mathrm{HU}$

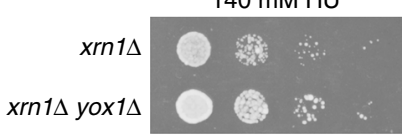

$175 \mathrm{mM} \mathrm{HU}$

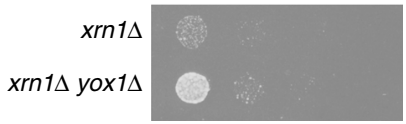

Fig. 4 YOX1 mRNA forms cytoplasmic granules and accumulates at P-bodies. a Representative images of cells co-expressing MS2-CP-GFP and Pat1-mCherry, with or without YOX1 tagged with MS2 repeats, before and after $2 \mathrm{~h}$ of treatment with HU. Cytoplasmic granules were assessed in both WT and in xrn1 $\Delta$ cells. Scale bars are $7 \mu \mathrm{m}$. Arrows indicate YOX1 mRNA granules that co-localize with Pat1-mCherry granules. b Quantification of YOX1 mRNA granules in untreated and $\mathrm{HU}$-treated cells. The average number of foci per cell is plotted for each of at least three replicates. The solid bar indicates the average of the replicates, $P$-values are from Student's $t$-tests, and $n$ indicates the total number of cells examined. c Serial 10-fold dilutions of the indicated strains were spotted on YPD with or without $\mathrm{HU}$. Plates were imaged after 3-4 days of growth to test the suppression of yox $1 \Delta \mathrm{HU}$ sensitivity by deletion of XRN1 
a

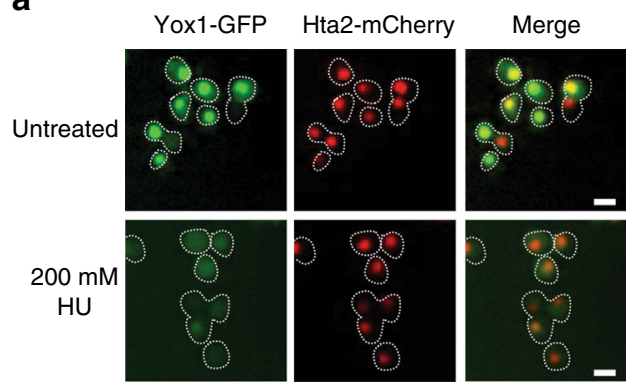

C

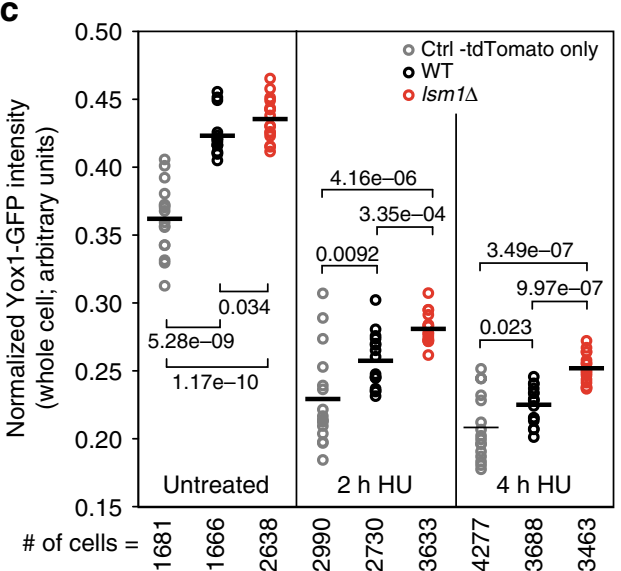

b

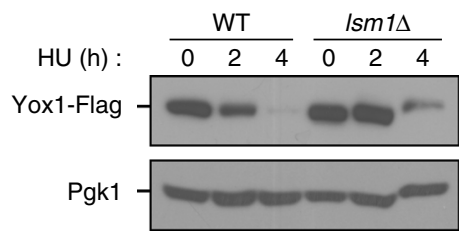

d

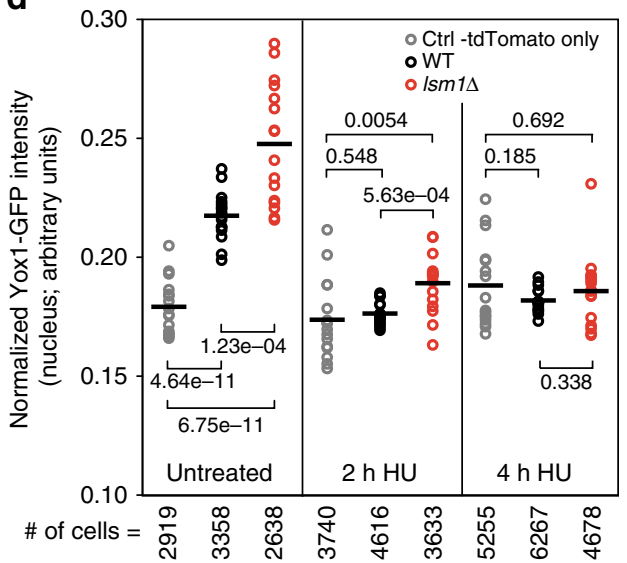

Fig. 5 The Yox1 repressor is more abundant in cells lacking LSM1. a Representative images of cells expressing Hta2-mCherry and Yox1-GFP before and after $2 \mathrm{~h}$ of HU treatment. The scale bars are $5 \mu \mathrm{m}$, and the outlines of the cells are indicated. $\mathbf{b}$ Analysis of Yox1-Flag protein level by immunoblot before and after HU treatment. Pgk1 was used as loading control. Molecular weight markers are shown on the uncropped image in Supplementary Fig. 5. One representative experiment is shown. Normalized intensity of the cellular (c) or the nuclear (d) Yox1-GFP fluorescence in WT and Ism1 $\Delta$ cells. Each point represents the data for an individual replicate of the indicated strain (for 8 technical replicates for each of 2 biological replicates). The solid bar indicates the average of the replicates, and $P$-values from the Student's $t$-test are indicated

results in the accumulation of non-functional P-bodies ${ }^{18}$, suggesting that YOX1 degradation is, to some extent, dependent on the formation of functional P-bodies during replication stress. However, because Lsm1-dependent and Pat1-dependent mRNA degradation can also occur in absence of visible P-body granules, it is possible that YOX1 mRNA regulation by $\mathrm{P}$-body proteins could occur at sites external to visible P-body granules.

We next tested whether YOX1 inactivation rescued the sensitivity of P-body mutants to diverse stresses or was specific to DNA replication stress induced by HU treatment (Fig. 3). Deletion of $L S M 1^{36}$ or PAT1 confers sensitivity to boric acid, yet deletion of YOX1 failed to rescue either sensitivity (Fig. 3a). The $l s m 1 \Delta$ mutant is also sensitive to heat stress ${ }^{37}$, and temperature sensitivity was not rescued by yox1 1 (Fig. 3a). The YOX1 gene has a paralog, $Y H P 1$, so we also assessed the effect of deleting $Y H P 1$ on stress resistance in $l s m 1 \Delta$ and pat $1 \Delta$ (Fig. 3a). Deletion of $Y H P 1$ had little effect on the HU, heat, or boric acid sensitivity of the P-body mutants. In addition, YHP1 mRNA was not upregulated in $l s m 1 \Delta$ (Supplementary Data 1). We conclude that deletion of YOX1 rescues the HU sensitivity of P-body mutants while playing little role in other P-body stress responses, and that the paralog YHP1 is unlikely to be a relevant P-body target during replication stress.

We reasoned that if P-body-dependent degradation of YOX1 mRNA is critical for replication stress resistance, then overexpression of YOX1 should sensitize P-body mutant strains to HU. Indeed, expression of YOX1 from the GAL1-10 promoter was toxic in both $l s m 1 \Delta$ and pat1 $\Delta$ backgrounds (Fig. 3b). Together these data support the conclusion that negative regulation of YOX1 expression by P-body proteins is important specifically for HU-stress resistance.

The YOX1 transcript localizes to P-bodies. To assess whether the negative regulation of YOX1 expression was likely occurring directly at the cytoplasmic P-body granules that form during replication stress, we tracked the location of YOX1 mRNAs in live cells using the mTAG mRNA visualization system ${ }^{38}$. We introduced MS2 repeats into the $3^{\prime}$ UTR of the YOX1 gene and expressed the MS2 coat protein fused to green fluorescent protein (GFP) to visualize the YOX1 mRNA. P-bodies were visualized simultaneously by co-expressing a Pat1-mCherry fusion protein. The MS2 tag system can compromise the visualization of fulllength mRNAs by promoting Xrn1-dependent accumulation of mRNA degradation products ${ }^{39,40}$, so we examined YOX1 mRNA localization in both WT and in cells deleted for XRN1. Additionally, we reasoned that because the YOX1 transcript is potentially degraded at P-bodies where $\mathrm{Xrn} 1$ is the predominant 5'-3' exoribonuclease ${ }^{41}$, deletion of XRN1 should facilitate YOX1 mRNA visualization by stabilizing it.

YOX1 mRNA forms granules in $\sim 6 \%$ of WT cells, both in untreated conditions and after $\mathrm{HU}$ treatment (Fig. 4a, b). Deletion of XRN1 dramatically increased the formation of YOX1 mRNA granules and of P-bodies ${ }^{18}$ in untreated conditions and after HU treatment (Fig. 4a, b). Control strains expressing the MS2-GFP coat protein but lacking the MS2 repeats in the 3UTR of YOX1 displayed very few granules (Fig. 4b), demonstrating that MS2-GFP focus formation required YOX1 tagged with MS2 
a

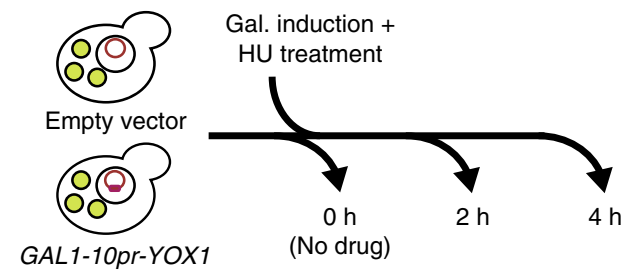

b

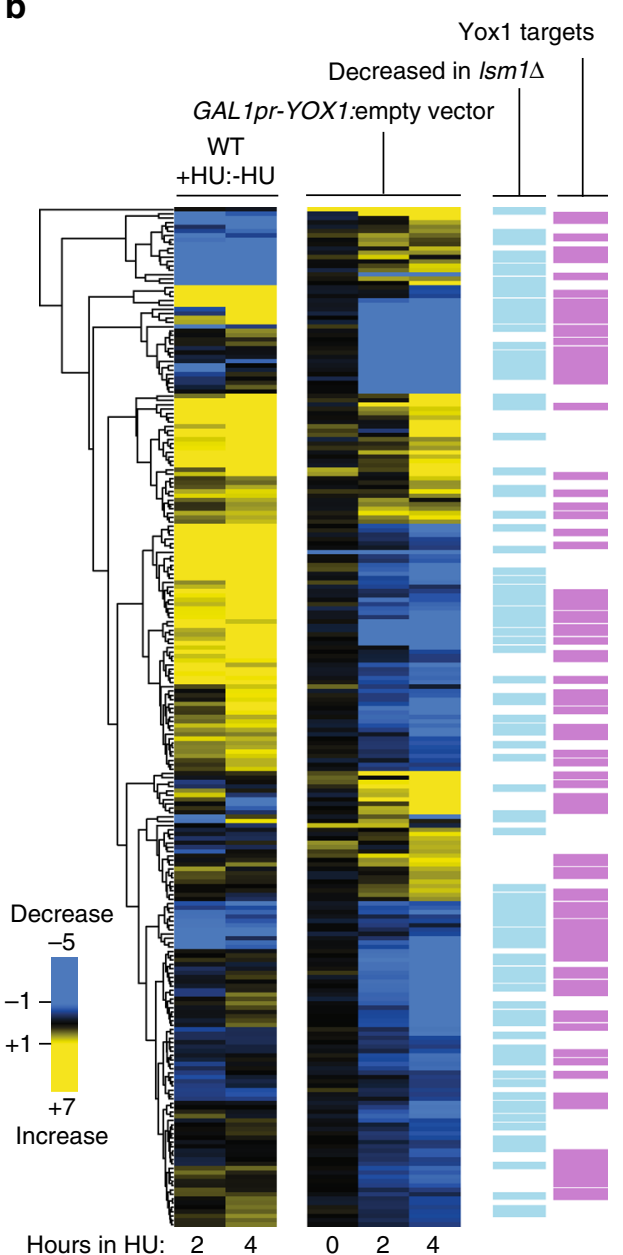

C

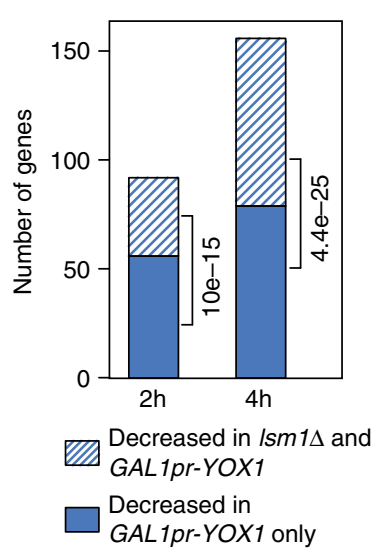

e

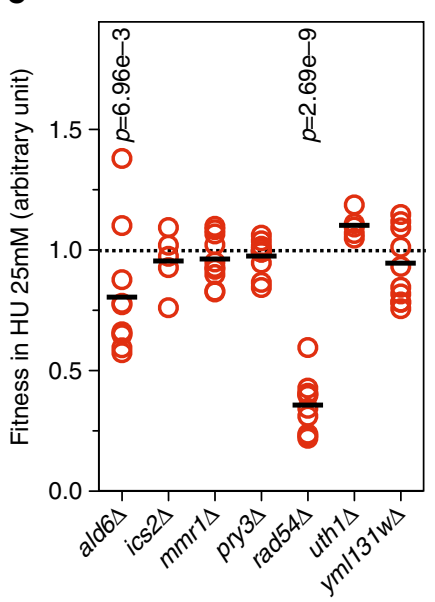

d

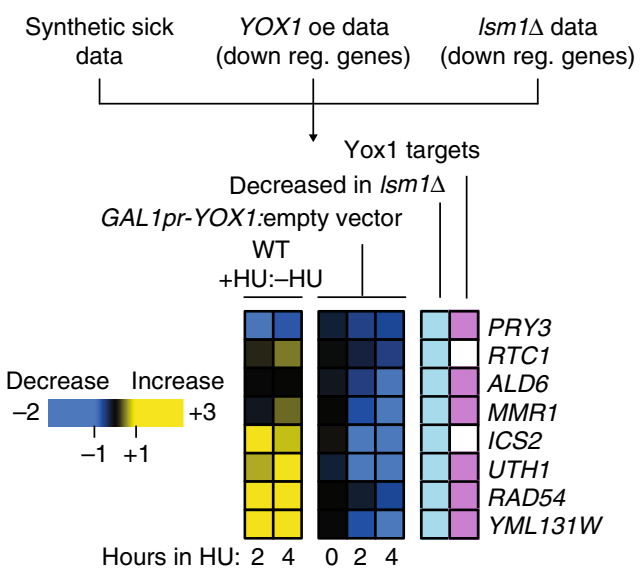

f

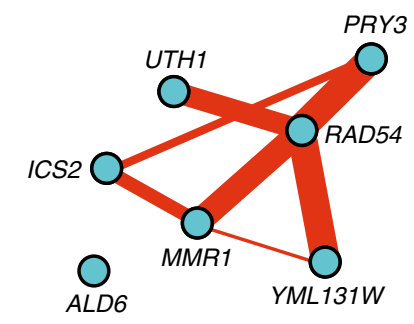

$-\mathrm{Gl}$ inducing $\mathrm{HU}$ sensitivity, $\mathrm{p} \leq 0.05$

-0.07 Fitness difference

-0.6 (HU minus untreated)

Fig. 6 Excess Yox1 represses genes that are critical for cell fitness during replication stress. a Overview of the RNA-seq experiment. WT cells containing pBY011 (empty vector) or pBY011-YOX1 (GAL1-10pr-YOX1) vectors were cultivated in synthetic media containing raffinose. Overexpression of YOX1 was induced by adding galactose and replication stress was induced by HU treatment. RNA was extracted at the indicated time points. $\mathbf{b}$ Heatmap of expression ratios generated by hierarchical clustering, showing genes that were differentially expressed upon YOX1 overexpression. Two different ratios are represented: $+\mathrm{HU}:-\mathrm{HU}$ compares expression values in the control strain (empty vector) between the HU condition and the untreated condition, and GAL1-10pr-YOX1:empty vector compares expression values between the YOX1-overexpressing strain and the control strain at each time point. Light blue bars indicate genes that were downregulated after $2 \mathrm{~h}$ and/or $4 \mathrm{~h}$ in $\mathrm{HU}$ in Ism1 $1 \Delta$ compared to WT (as in Fig. 1). Purple bars denote genes that are described as Yox1 targets in the Yeastract database. $\mathbf{c}$ The number of genes with decreased mRNA abundance upon YOX1 overexpression, with the overlap of genes that also have decreased mRNA abundance in $I s m 1 \Delta$ is plotted as a stacked bar graph. The indicated $P$-values were calculated using a hypergeometric test. d Identification of potential Yox1 targets during replication stress at the intersection of three datasets. Heatmaps are as presented in $\mathbf{b}$. e Fitness data in $\mathrm{HU}$ for the single mutants identified in $\mathbf{d}$. Each data point represents a single replicate $(n=5)$. Horizontal bars represent the average of the replicates. $P$-values are from a Student's t-test. $\mathbf{f}$ Condition-dependent negative genetic network of interactions between the 8 targets in d. Red edge thickness is proportional to the difference between the double mutant fitness in absence and presence of $25 \mathrm{mM} \mathrm{HU}$

repeats. Importantly, the $Y O X 1$ granules co-localized with the $\mathrm{P}$ body marker Pat1 in both WT and $x r n 1 \Delta$ cells under normal conditions and after HU treatment (Fig. 4a, b). In 1092 YOX1 granules examined, $25.8 \pm 8.8 \%$ of YOX1 mRNA and Pat1 granules co-localized in $x r n 1 \Delta$ untreated cells. Similar levels of co-localization were found with other transcripts and the P-body marker Edc $3^{42}$. HU treatment resulted in the appearance of large aggregates in the red channel, making the quantification of $Y O X 1$ mRNA granule co-localization with Pat1-mCherry difficult to evaluate (Fig. 4a). Yet, when discreet Pat1 foci were observable we could detect co-localization with YOX1 mRNA granules (Fig. 4a). Interestingly, the accumulation of YOX1 mRNA granules that is 
evident upon deletion of XRN1 correlates with increased HU sensitivity (Fig. 4c) and increased YOX1 transcript abundance $(x r n 1 \Delta$ : wildtype $=3.7 \pm 1.8)$. As was the case with $l s m 1 \Delta$, the HU sensitivity of $x \mathrm{rn} 1 \Delta$ is rescued by deletion of YOX1 (Fig. 4c), indicating that YOX1 mRNA is a critical transcript for $\mathrm{HU}$ sensitivity of $x r n 1 \Delta$. Thus, failure to degrade YOX1 mRNA, either at the level of functional P-body formation or at the level of nucleolytic degradation of the mRNA, results in HU sensitivity. These data reinforce the notion that control of

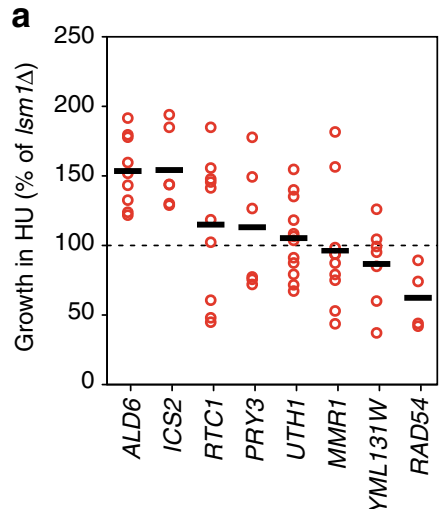

b
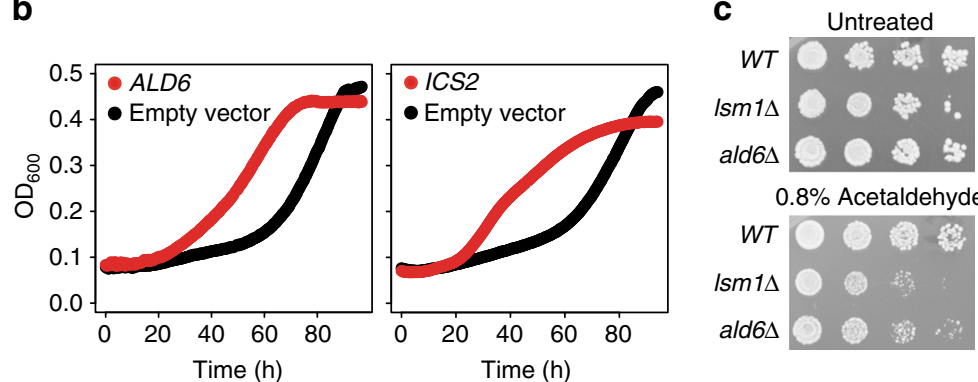

d
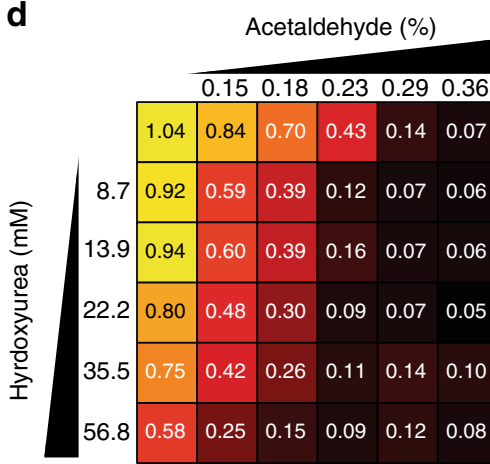

Fitness: 0

$\mathbf{f}$

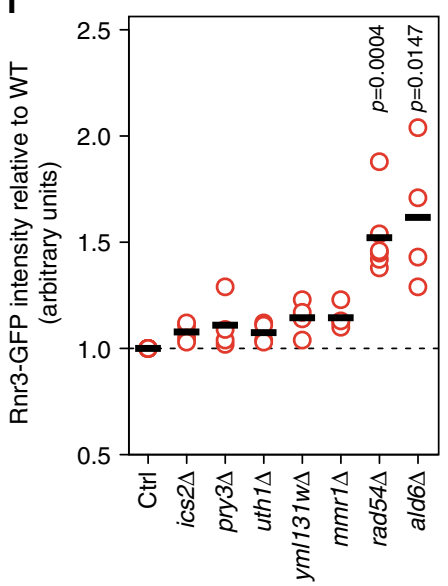

Acetaldehyde (\%)

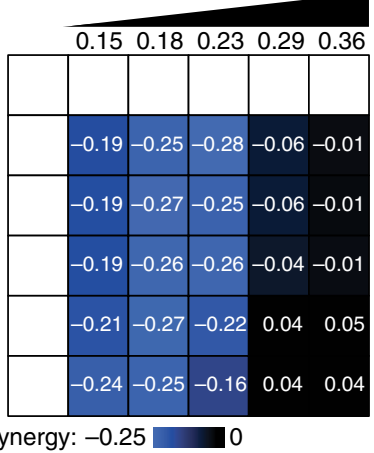

$\mathbf{9}$
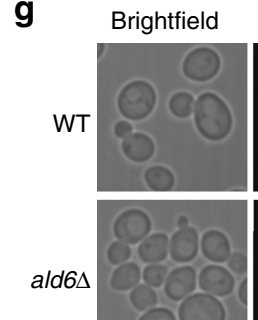

Rnr3-GFP Rpl39-tdTomato
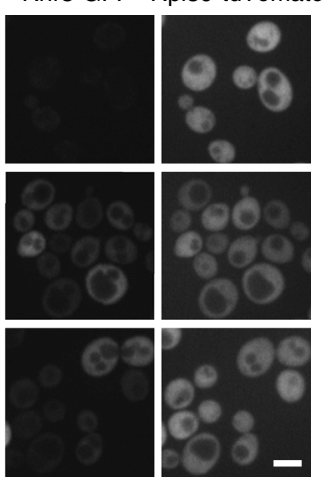

$\operatorname{rad} 54 \Delta$

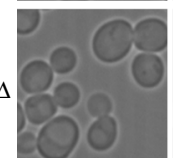

e

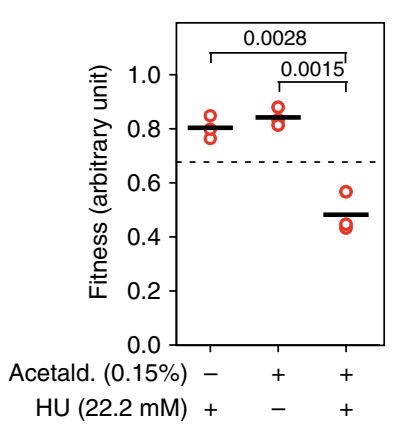

h

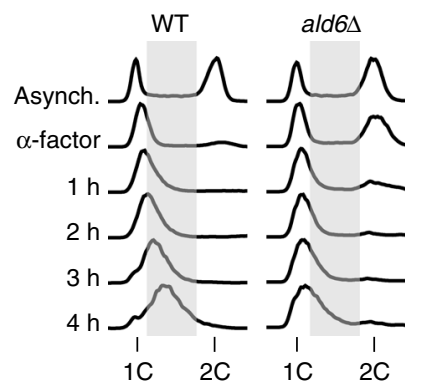

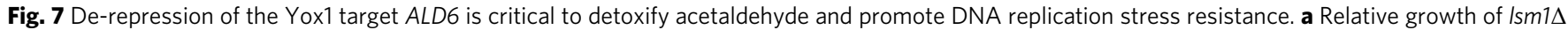
cells overexpressing the indicated genes compared to $1 \mathrm{sm} 1 \Delta$ cells containing the control vector. Each data point represents a single replicate. Each experiment was repeated at least three times and included two independent biological replicates each time. The solid bar indicates the average. $\mathbf{b}$ Growth curves of Ism1 $1 \Delta$ cells overexpressing ALD6 or ICS2 (red) or Ism1 cells containing the control vector (black) in the presence of HU. c Serial 10-fold dilutions of the indicated strains were spotted on YPD with or without acetaldehyde. Plates were imaged after 3-4 days of growth. $\mathbf{d}$ Left: Heatmap of fitness values of cells grown in presence of acetaldehyde, hydroxyurea or combinations of both drugs. Fitness values are relative to the untreated condition, for three independent replicates. Right: Synergy heatmap. Indicated values correspond to the difference between the observed fitness in the given drug combination and the expected fitness. e Fitness of cells grown in presence of acetaldehyde, hydroxyurea or combination of both drugs at the indicated concentrations. Each data point represents one replicate. P-values are from a Student's $t$-test. $\mathbf{f}$ Rnr3-GFP intensities in the indicated mutants relative to the WT. Each data point represents one replicate. $P$-values are from a Student's $t$-test. $\mathbf{g}$ Representative microscopic images of the indicated strains expressing Rnr3-GFP and RPL39pr-TdTomato. Scale bar: $5 \mu \mathrm{m}$. h Cell-cycle progression of the WT and ald6 $\Delta$ strain after alpha-factor block and release in YPD containing $200 \mathrm{mM}$ of $\mathrm{HU}$ 
YOX1 mRNA abundance is important to the replication stress response.

Together, these data indicate that YOX1 mRNAs localize to P-bodies both in normal growth and during replication stress induced by $\mathrm{HU}$, consistent with a model in which YOX1 mRNA abundance is regulated by $\mathrm{P}$-body proteins, including $\mathrm{Xrn} 1$, directly at P-bodies.

Yox1 accumulates in the nucleus in $l s m 1 \Delta$ cells. Yox 1 and its paralog Yhp1 bind to the transcriptional antirepressor $\mathrm{Mcm} 1$, resulting in repression of $\mathrm{Mcm} 1$ targets $^{33}$. Having established that the YOX 1 transcript increases in $l s m 1 \Delta$ cells in the presence of HU (Fig. 2c), we tested whether the Yoxl protein is increased in abundance as well. Yox1 accumulates in the cytoplasm upon HU treatment $^{8}$, so we examined Yoxl-GFP protein abundance in individual cells and in individual nuclei in both WT and $l s m 1 \Delta$ cells (Fig. 5). Cells lacking LSM1 had elevated levels of Yox1-GFP, in the nucleus and in the cytoplasm (Fig. 5c, d), consistent with the increased YOX $1 \mathrm{mRNA}$ abundance in $l s m 1 \Delta$. The increased level of Yox1-GFP in the nucleus in $l s m 1 \Delta$ persisted upon HU treatment for at least $2 \mathrm{~h}$ (Fig. 5d). Immunoblot analysis of Flagtagged Yox 1 confirmed an increase of Yox 1 protein in $l s m 1 \Delta$ cells (Fig. 5b). It is worth noting that Yox1 protein abundance did not precisely mirror YOX1 mRNA abundance, suggesting additional regulation at the level of translation and/or protein stability. These data indicate that there is more Yoxl in the nucleus when the YOX1 mRNA is not degraded effectively at P-bodies, and suggest that Yox1 target genes will be expressed at lower levels when P-body function is compromised.

The network of Yoxl targets is important for $\mathrm{HU}$ resistance. To probe the consequences of increased YOX1 expression during replication stress, we used RNA-seq to measure transcript abundance during YOX1 overexpression in the presence of $\mathrm{HU}$ (Fig. 6a, b and Supplementary Data 4). The expression of between 96 (at $2 \mathrm{~h}$ ) and 156 genes (at $4 \mathrm{~h}$ ) was decreased in the YOX1overexpressing strain. One third $(2 \mathrm{~h})$ to one half $(4 \mathrm{~h})$ of the genes that were downregulated upon YOX1 overexpression were also downregulated in the $l s m 1 \Delta$ cells, consistent with modulation of Yox1 function by Lsm1 (Fig. 6b, c). This effect is statistically supported as $l s m 1 \Delta$ downregulated genes were strongly enriched among the downregulated genes identified upon YOX1 overexpression (Fig. 6c). Interestingly, a number of the genes with altered expression in the presence of HU and YOX1 overexpression were not known Yox1 targets ${ }^{43}$ (Fig. 6b, purple panel). Therefore the potential repertoire of Yox1 regulatory targets is expanded during replication stress.

Surprisingly, the YOX1 transcriptional repression program that we identified was not enriched for genes involved individually in HU resistance (hypergeometric $P=0.992$ at $2 \mathrm{~h}$ and $P=0.998$ at $4 \mathrm{~h}$ ). We focussed on the 8 Yoxl targets that were also downregulated in $l s m 1 \Delta$ and that caused HU synthetic sickness in $l s m 1 \Delta$ (Fig. $6 \mathrm{~d}$ ), reasoning that the most critical Yox1 targets would be affected by Yox1 overexpression, LSM1 deletion, and would exacerbate the HU sensitivity of $l s m 1 \Delta$. We noted that only two (ALD6 and RAD54) were sensitive to DNA replication stress on their own (Fig. 6e). To test the possibility that redundancy could account for the relative absence of singlemutant HU sensitivity, we constructed 20 combinations of double knockout mutants of the 8 targets (excluding RTC1 which was not available in our deletion collection). We analyzed fitness by monitoring growth in liquid medium in presence or absence of HU. Using this approach, we identified 7 negative genetic interactions that were worsened by the presence of DNA replication stress (Fig. 6f, Supplementary Data 5). Three interactions involved genes that were not HU sensitive as single mutants, and four involved RAD54. Therefore, functional redundancy among genes in the YOX1 regulome can mask the roles of individual genes in replication stress resistance. We speculate that detailed analysis of double mutant space in the YOX1 regulome would reveal many additional interactions that confer HU sensitivity.

Together, these data suggest that accumulation of Yox1 that occurs in the absence of a functional P-body pathway results in the downregulation of a transcriptional network which as a whole maintains cell fitness during replication stress.

Acetaldehyde detoxification is critical upon HU exposure. Although the YOX1 transcriptional program as a whole is likely to contribute to replication stress resistance, we also tested whether we could detect roles for individual target genes. The 8 identified targets are downregulated upon YOX1 overexpression and when P-body function is compromised (Fig. 6d). Therefore, we expressed each gene from the GAL1-10 promoter in an $l s m 1 \Delta$ strain in the presence of $\mathrm{HU}$ to test whether any could individually rescue the HU sensitivity of $l s m 1 \Delta$ (Fig. 7a, b). Expression of ICS 2 or ALD6 increased the fitness of $l s m 1 \Delta$ during replication stress (Fig. 7a, b) indicating that de-repression of ICS2 and ALD6 via P-body protein dependent degradation of YOX1 mRNA contributes to replication stress resistance. We identified binding sites for both Yox1 and its co-repressor Mcml in the 1000-bp promoter regions of ALD6 and ICS2 using YeTFaSCo ${ }^{44}$ (Supplementary Table 3), although it is also possible that both are indirect targets. Finally, $l s m 1 \Delta$ is sensitive to acetaldehyde (Fig. 7c), as would be expected if expression of the aldehyde dehydrogenase encoded by ALD6 is reduced when P-bodies are not functional.

We probed the mechanism by which ALD6 contributes to replication stress resistance. First we used quantitative RT-PCR to confirm that ALD6 mRNA abundance decreases in $l s m 1 \Delta$ (Supplementary Fig. 6). Ald6 converts acetaldehyde into acetate, and thus ald $6 \Delta$ cells accumulate higher amounts of acetaldehyde ${ }^{45}$. Acetaldehyde induces DNA inter-strand crosslinks that block DNA replication and transcription ${ }^{46}$ and therefore could potentially be toxic in cells experiencing DNA replication stress. We found that $\mathrm{HU}$ and acetaldehyde were synergistic over a range of concentrations, indicating that the presence of acetaldehyde during $\mathrm{HU}$-induced replication stress is indeed toxic (Fig. 7d, e). Deletion of ALD6 causes strong constitutive Sphase checkpoint activation, as revealed by the induction of RNR3 expression in ald $6 \Delta$ cells to similar level as in rad54 $\Delta$, one of the strongest inducers of checkpoint-dependent RNR3 expression ${ }^{15}$ (Fig. 7f, g). Consistent with the accumulation of replication blocking DNA lesions in cells lacking Ald6, and the synergy between acetaldehyde and $\mathrm{HU}$, we found that ald $6 \Delta$ cells displayed slow S-phase progression in HU relative to WT cells.

Together, these data suggest that acetaldehyde accumulation causes endogenous replication stress that sensitizes cells experiencing additional sources of DNA replication stress. Therefore, de-repression of ALD6 during HU-induced replication stress promotes fitness by removing acetaldehyde, reducing DNA damage, and reducing barriers to DNA synthesis.

\section{Discussion}

P-bodies form in the cytoplasm in response to a wide range of stresses, including DNA replication stress induced by $\mathrm{HU}^{8}, 14$. Several lines of evidence indicate that P-body function is important for the cellular replication stress response: At least two genes regulate P-body formation in replication stress but not in other stresses ${ }^{8}$. Deletion of P-body genes confers sensitivity to 


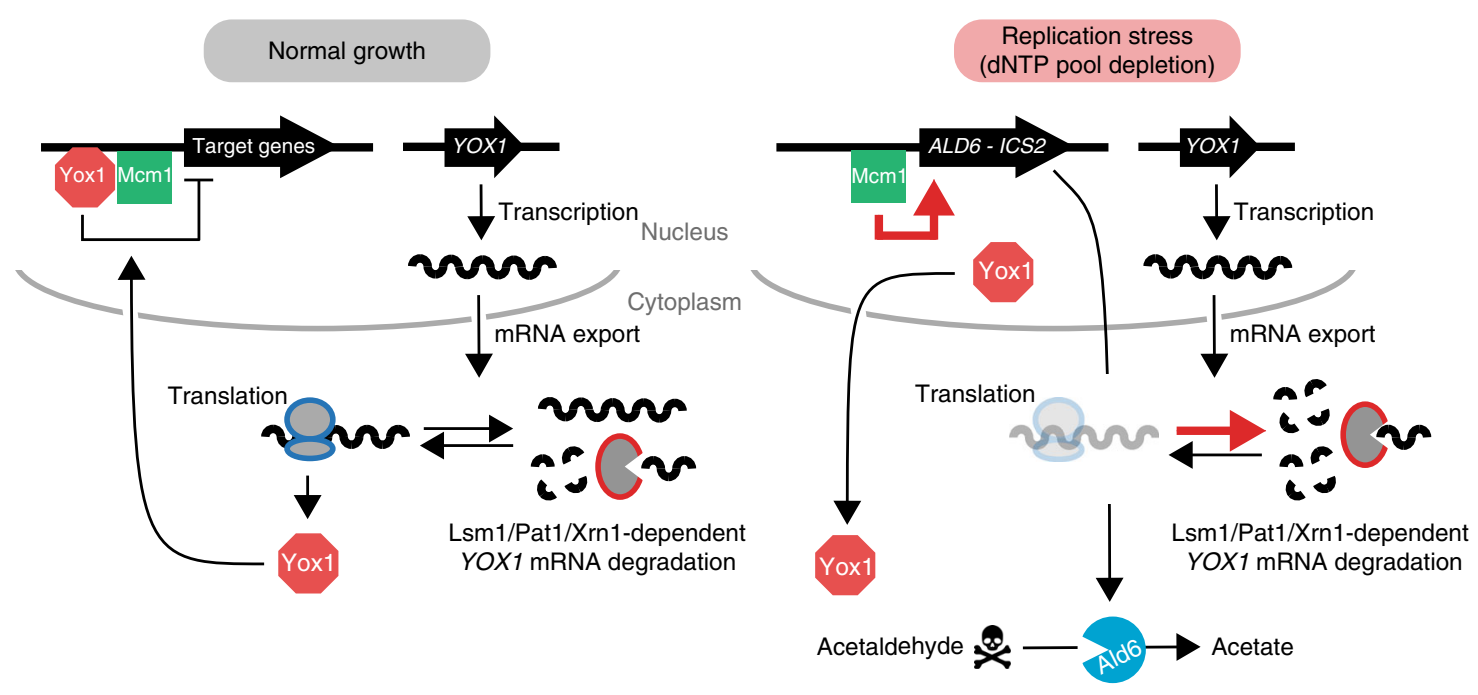

Fig. $8 \mathrm{~A}$ model for YOX1 post-transcriptional regulation by P-bodies during replication stress. During normal growth YOX1 mRNA is produced and exported to the cytoplasm where it can either be degraded or translated. YOX1 mRNA translation produces a transcriptional repressor that is imported to the nucleus where it binds the $\mathrm{Mcm} 1$ antirepressor to represses the transcription of target genes. During replication stress induced by hydroxyurea treatment, $\mathrm{P}$-body formation is increased which results in increased YOX1 mRNA degradation (red arrow). Export of the Yox1 protein from the nucleus concomitant with degradation of the YOX1 mRNA allows the cells to control the amount of the Yox1 repressor in the nucleus. Decreased nuclear Yox 1 relieves transcriptional repression (red arrow) of target genes required for $\mathrm{HU}$ resistance, including ALD6 and ICS2. De-repression of ALD6 promotes degradation of acetaldehyde, a molecule that is strongly toxic during replication stress

replication stress ${ }^{8,} 32$. One biologically relevant P-body target in $\mathrm{HU}$, the gene encoding histone $\mathrm{H} 3$, has been identified ${ }^{32}$. Finally, P-body mutants display spontaneous DNA replication stress ${ }^{15,32}$. Here we defined the P-body-regulated transcriptome, both during normal growth and in the presence of replication stress. We leveraged the RNA abundance data to identify five mRNA transcripts whose P-body regulation is important for replication stress resistance, ARL3, ACF4, RRS1, TMA16 and YOX1. We demonstrated that YOX1 upregulation is toxic in cells lacking a functional P-body pathway, and that YOX1 mRNA localizes to P-bodies in live cells, consistent with direct degradation of YOX1 mRNA at P-bodies. Finally, we showed that in the absence of proper regulation of YOX1 mRNA by P-body proteins, Yox1 protein accumulates in the nucleus resulting in aberrant regulation of gene expression during replication stress. We propose a model (Fig. 8) where P-body formation is induced in response to replication stress, providing the cell with increased mRNA degradation capacity, resulting in a reduced pool of YOX1 mRNA available for translation. Increased YOX1 mRNA degradation, concomitant with the export of the Yoxl protein from the nucleus precludes the nuclear accumulation of the Yoxl repressor during replication stress. This orchestrated response allows cells to de-repress a program of gene expression, and individual genes, needed for replication stress survival.

Since mRNA degradation can occur in the absence of visible P-bodies ${ }^{47,48}$, it is possible that assembly of P-body proteins into microscopically visible structures is not required to regulate transcript abundance during replication stress. However, we find that at least three P-body proteins (Lsm1, Pat1, and Xrn1) regulate YOX1 mRNA abundance. Loss of Lsm1 triggers accumulation of non-functional P-bodies in unstressed cells, as does loss of Xrn1, and prevents Pat1 recruitment to P-bodies during glucose starvation stress ${ }^{18}$. Loss of Pat 1 also prevents functional P-body formation during DNA replication stress induced by $\mathrm{HU}^{8}$. In addition, we observed accumulation of YOX1 mRNA cytoplasmic granules upon Xrn1 deletion, and many of the YOX1 mRNA granules contained Pat1. Although we cannot exclude the possibility that regulation of YOX1 mRNA by P-body proteins occurs in the soluble fraction of the cytoplasm, our data are consistent with a model in which at least a portion of YOX1 mRNA is degraded at P-bodies.

As expected, we identified a group of transcripts whose abundance increases in $l s m 1 \Delta$ cells, consistent with the possibility that some are degraded at P-bodies in WT cells. Surprisingly, we found an almost equal number of mRNAs whose abundance decreases in $l s m 1 \Delta$ cells. Interestingly, this paradoxical effect has been described in studies exploring the interconnection between transcription and mRNA degradation. For instance, the cytoplasmic mRNA exonuclease Xrn1 buffers transcription by activating the Nrg1 transcriptional repressor ${ }^{21}$. Although Nrg1 was not upregulated in $l s m 1 \Delta$ cells, the upregulation of the Yox1 repressor in $l s m 1 \Delta$ that we observe explains a fraction of the downregulated genes ( $5 \%)$. Upregulation of other transcriptional repressors could further increase the number of genes downregulated in $l s m 1 \Delta$, although we did not identify additional repressors in our dataset. Another possibility is that transcriptional activators are downregulated in $l \operatorname{sm} 1 \Delta$ cells and therefore fail to activate their targets. We did find cases of downregulated transcriptional activators (Fkh2, Bas1, Rgt1, Pho2 and Leu3) in cells lacking Lsm1, and also found that the targets of these activators were enriched in the set of $l \operatorname{sm} 1 \Delta$ downregulated genes (average hypergeometric $P=2.7 \times 10^{-3}$ at $4 \mathrm{~h}$ in $\mathrm{HU}$ ). Together, this phenomenon could account for an additional $\sim 5 \%$ of the downregulated genes in $l s m 1 \Delta$ cells. Interestingly, mRNA degradation factors such as Xrn1, Dcp2, and Lsm1 can shuttle between the cytoplasm and the nucleus where they bind to gene promoter regions and stimulate transcription initiation and elongation ${ }^{49}$, raising the further possibility that some fraction of the downregulated $l s m 1 \Delta$ genes are direct nuclear targets of Lsm1. Finally, given the magnitude of the transcriptional downregulation in the absence of LSM1 it is possible that P-bodies can in some instances protect and stabilize mRNAs from exosomedependent degradation ${ }^{20}$, perhaps in concert with translational repression ${ }^{50}$.

We find that LSM1 is critical for the post-transcriptional regulation of mRNA abundance during replication stress. Exposure 
to HU-induced replication stress causes massive mRNA abundance perturbations (this study and Dubacq et al. ${ }^{6}$ ), between $25 \%$ ( $1 \mathrm{~h} \mathrm{HU}$ ) and $45 \%$ ( $4 \mathrm{~h} \mathrm{HU}$ ) of which involve Lsm1 (and likely P-body) function. Of the mRNAs whose abundance was altered in a P-body-dependent manner during HU treatment (HU-responsive genes), only $11.8 \%$ were general environmental stress response genes, indicating that LSM1 regulates much of the specific mRNA abundance response to replication stress. In total, combining deletion of LSM1 with replication stress resulted in the accumulation of between 499 and 1203 transcripts. Distinct biological processes were enriched in the set of transcripts that accumulated in $l s m 1 \Delta$ during replication stress, suggesting that the response was specific and biologically significant. Although the primary target of $\mathrm{HU}$ is ribonucleotide reductase, inactivation of which limits dNTP pools to cause replication stress, there is evidence that $\mathrm{HU}$ affects other processes including iron metabolism $^{51,52}$. We have no doubt that some fraction of the $l s m 1 \Delta$-affected HU transcriptome could reflect cellular events in addition to replication stress. However, we found that between 25 and $53 \%$ of affected genes (including YOX1) overlap with genes that are differentially expressed in a distinct replication stress agent, MMS, and that at least two of the effectors of the Yox1 transcriptional program, $R A D 54$ and $A L D 6$, are individually important to prevent DNA replication stress. We conclude that the key elements of the $l s m 1 \Delta$-affected HU transcriptome that we have deciphered are relevant to DNA replication stress. The functional targets of Lsm1 and P-bodies in other forms of stress, both chemical and genetic, remain to be determined.

In addition to the P-body-dependent regulation of YOX1 and HHT1 mRNAs, we identified 4 other P-body targets during replication stress: ACF4, ARL3, TMA16 and RRS1. The P-bodydependent regulation of these genes is likely to contribute significantly to $\mathrm{HU}$ resistance, as their inactivation results in suppression of the HU sensitivity of P-body mutants comparable to that seen upon deletion of YOX1 or HHT1. Of particular interest, two of the putative P-body targets have ribosomal functions. RRS1 encodes an essential protein that binds to the $60 \mathrm{~S}$ ribosomal subunit and is also required during ribosome biosynthesis ${ }^{29}$. TMA16 encodes a protein of unknown function that associates with ribosomes ${ }^{30}$. We previously demonstrated that Asc1, the ortholog of the human RACK1 translation inhibitor, promotes P-body formation during replication stress ${ }^{8}$, further connecting P-bodies, DNA replication stress, and protein translation.

We also find a cohort of genes that when deleted suppress the HU sensitivity of P-body mutants, but that do not appear to be regulated by P-bodies at the level of transcript abundance. These mRNAs might represent targets of translational repression, rather than degradation, at P-bodies. Several lines of evidence connect regulation of translation to the DNA damage response. Translational repression of the tumor suppressor p53 can be mediated by the long noncoding RNA RoR during DNA damage ${ }^{53}$ and by the $\mathrm{Ku}$ DNA-binding protein in unperturbed cells ${ }^{54}$. UV damage, ionizing radiation, and topoisomerase inhibition each result in a general inhibition of translation accompanied by selective translation of DNA damage response mRNAs ${ }^{55-57}$. Direct links between replication stress and protein translation are currently sparse, although levels of the MCM helicase are downregulated post-transcriptionally by replication stress ${ }^{58}$. It is clear, however, that extensive remodeling of protein abundance occurs during replication stress, and the majority of protein abundance changes are not explained by changes in mRNA abundance ${ }^{8,9}$. Together, these observations raise the possibility that cellular translation capacity is regulated by $\mathrm{P}$-body proteins during DNA replication stress.
Regulation of expression of the Yox1 transcriptional repressor is critical during the replication stress response. Overexpression of YOX1 resulted in the downregulation of genes that are normally induced by HU treatment, suggesting that de-repression of Yox1 targets forms a large component of the transcriptional response to replication stress. Deletion of YOX1 rescues the HU sensitivity of P-body mutants $(l s m 1 \Delta$, pat $1 \Delta$ and $x r n 1 \Delta)$. The simplest explanation for this rescue is that $Y O X 1$ gene expression is excessive when P-bodies are dysfunctional, indicating that de-repression of YOX1 targets is critical for cell fitness during replication stress. Surprisingly, we did not find many genes that are individually involved in $\mathrm{HU}$ resistance within the Yox1 transcriptional network. This observation led us to test whether the Yox1 regulon as a whole could exert an important role in HU resistance. Although we tested only a small subset of Yox1 targets, we readily detected double mutant combinations that cause HU sensitivity in the Yox1 transcriptional network, consistent with the idea that de-repression of more than one Yoxl target is required to maximize cell fitness during HU-stress. This possibility has been noted repeatedly in different DNA damage and replication stress-induced transcriptomes, where the overlap between individual regulated genes and genes that confer stress sensitivity when deleted individually is typically poor ${ }^{4,5,59}$.

In addition to the role that the entire Yox1 regulon might exert to promote $\mathrm{HU}$ resistance, we also identified individual YOX1 targets whose de-repression is critical to avoid replication stressinduced toxicity. In particular, ALD6 encodes a key enzyme involved in acetaldehyde detoxification both under normal growth and during stress conditions ${ }^{60}$. Acetaldehyde can induce DNA inter-strand crosslinks that block DNA replication and transcription $^{46}$ and we found that acetaldehyde accumulation induces replication stress and greatly sensitizes cells to additional DNA replication stress induced HU. Interestingly, DNA interstrand crosslink repair is essential for the survival of human cells experiencing endogenous replication stress ${ }^{61}$ and aldehyde dehydrogenases like Ald6 are conserved throughout evolution, suggesting that accumulation of acetaldehyde could be an important contributor to DNA replication stress in all cells. Thus, the Yox1 regulon contributes to HU resistance both at the level of individual genes, and at the level of higher-order combinations of genes, consistent with transcriptional re-wiring in the absence of P-body proteins being an important element of the response to HU-induced DNA replication stress.

\section{Methods}

Yeast strains and growth conditions. All strains used in this study are listed in Supplementary Table 4. yox $1 \Delta, y h p 1 \Delta, x r n 1 \Delta$, and ald $6 \Delta$ strains were obtained from the yeast deletion collection ${ }^{62}$. Yeast were maintained on standard YPD or synthetic complete drop-out medium lacking the appropriate amino acids when necessary and grown at $30^{\circ} \mathrm{C}$ unless specified otherwise. For overexpression experiments, yeasts were first grown to saturation in synthetic drop-out medium containing $2 \%$ raffinose as the only carbon and then diluted in fresh medium containing raffinose and $2 \%$ galactose, unless specified otherwise. All yeast strains were constructed in BY4741 or BY4742 backgrounds ${ }^{63}$ using standard lithium acetate transformation. Strains expressing $Y O X 1$ tagged with MS2 repeats were constructed as described ${ }^{38}$.

RNA extraction and sequencing for wild type and $\mid s m 1 \Delta$. WT and $l s m 1 \Delta$ (JTY16) cells were grown to mid-logarithmic phase in YPD, sampled, and then treated with $200 \mathrm{mM} \mathrm{HU}$, and sampled after 1, 2 and $4 \mathrm{~h}$. Approximately $20 \mathrm{OD}_{600}$ of cell culture was used for RNA extraction. RNA extractions were performed using the yeast RiboPure kit (ThermoFisher Scientific) according to the manufacturer's instructions. Prior to sequencing library preparation, rRNAs were removed from the RNA samples using the yeast RiboZero kit (Illumina). Sequencing libraries were prepared using the TruSeq RNA Library Prep v2 kit (Illumina) and sequenced on a HiSeq2500 using v4 chemistry (Illumina). RNA-sequencing data were analyzed using the Tuxedo protocol ${ }^{19}$. Briefly, the $50 \mathrm{bp}$ reads were aligned to the sacCer3 version of the $S$. cerevisiae genome with Tophat $2^{64}$ using the default parameters. Between 15 and 20 millions reads were obtained for every sample and alignment to the reference genome was achieved for more than $95 \%$ of the reads for every 
sample. Genome annotations were downloaded from the SGD database (http:// www.yeastgenome.org) on 7 October 2014. Transcripts were assembled with Cufflinks ${ }^{19}$ and merged assembly files were generated with Cuffmerge ${ }^{19}$. Differential expression was assessed using Cuffdiff ${ }^{19}$. Default parameters were used to run Cufflinks, Cuffmerge, and Cuffdiff. Log2 ratios and statistical data generated with Cuffdiff were further analyzed using custom pipelines in R (https://www. r-project.org). Mean FPKM values, $\log 2$ ratios and $P$-values for every experiment are provided in Supplementary Data 1. Each RNA-sequencing experiment included two independent technical replicates. Correlation between replicates was always greater than 0.92. Raw sequencing data are accessible on GEO Omnibus with the accession number GSE83515. R scripts are provided in Supplementary Software 1.

To validate the results obtained using the Tuxedo analysis pipeline, RNA-Seq data were re-analyzed using $\mathrm{EBSeq}^{23}$ or edge $\mathrm{R}^{24}$. Raw sequencing count data generated by Cuffdiff before conversion to FPKM values were extracted for each gene and each RNA-Seq sample to create a matrix of raw counts compatible with EBSeq or edgeR analysis (Supplementary Data 1). EBSeq and edgeR analysis were performed using the respective R packages (EBSeq v1.16.0 and edgeR v3.18.1) downloaded from the Bioconductor website and according to the manual instructions.

Protein and genetic interactions enrichment analysis. Protein-protein and genetic interaction data were downloaded from BioGRID (http://thebiogrid.org/) on 24 August 2016. For each gene in the BioGRID data, a list of non-redundan interactors was generated. For each set of differentially expressed genes, the number of interactions involving all the genes in the given gene set (untreated or HU-core and increase or decrease in abundance) was determined and normalized to the number of genes in the given gene set. The same calculation was performed on the entire BioGRID dataset. $P$-values were calculated using the binomial test in $\mathrm{R}$.

GO term analysis. GO term analysis was performed using the GO term finder tool (http://go.princeton.edu/) using a $P$-value cutoff of 0.01 and applying Bonferroni correction, querying biological process enrichment for each gene set. Transposon genes were removed from the analysis due to their repetitive nature. GO term enrichment results were further processed with REViGO ${ }^{65}$ using the "Medium $(0.7)$ " term similarity filter and simRel score as the semantic similarity measure. GO term network data generated by REViGO was loaded into Cytoscape v3.2.0.6 for visualization as shown in Fig. 1. Nodes were re-organized for clarity, and nodes representing terms with a frequency greater than $15 \%$ in the REViGO output were eliminated as too general. Gene lists used for the GO enrichment analyses, as well as the list of enriched GO terms obtained are provided in Supplementary Data 2.

Synthetic genetic array-based suppressor screen. SGA procedure: Strains RLKY6 (lsm1 $1 \Delta$ ) and RLKY15 (pat1 $\Delta$ ) (Supplementary Table 1) were crossed to the yeast deletion collection ${ }^{62}$ and a collection of temperature sensitive mutants for a set of essential genes ${ }^{27}$ in a condensed 1536 colony array format using a BioMatrix (S\&P Robotics Inc.) automated pinning system. In this format, each mutant on the deletion and temperature sensitive arrays gave rise to one diploid colony for each query strain. After diploid selection, yeast diploid strains were sporulated and MATa haploid double-mutants were selected using standard SGA media ${ }^{28}$. After double mutant selection, the condensed 1536 array was expanded to a quadruplicated 1536 array (four colonies for each double mutant) and pinned on double mutant selection SGA medium once again. To identify suppressors of $l s m 1 \Delta$ and pat1 $\triangle \mathrm{HU}$ sensitivity, the quadruplicated 1536 arrays were first pinned on double mutant selection SGA medium. The newly pinned arrays were then immediately used as pinning source to re-pin the cells on double mutant selection SGA medium containing $\mathrm{HU}$ at concentrations of 100 and $150 \mathrm{mM}$ for the deletion array and temperature sensitive array, respectively. Cells were grown for 3 days at $30^{\circ} \mathrm{C}$. Plates were photographed in order to determine the colony size computationally. The experiment was repeated three times, with the exception of pat $1 \Delta \times x x x$ ts, which was repeated twice.

Calculation of fitness in $\mathrm{HU}$ : We defined the fitness in $\mathrm{HU}$ as the ratio between the colony size in $\mathrm{HU}$ and the colony size in absence of drug. Raw colony size for each double mutant grown in absence or presence of $\mathrm{HU}$ was determined using SGATools ${ }^{67}$ (http://sgatools.ccbr.utoronto.ca/) and further data analysis was perform in R using a customized pipeline. Double mutants displaying small colony size in the absence of drug were removed from the analysis. For each of the remaining double mutants, fitness in HU was calculated and normalized on a per plate basis using the $Z$-score method (difference between the colony size of a given mutant and the average colony size on the plate divided by the standard deviation observed for colony size on the plate). All mutants with an average $Z$-score, between three replicates, greater than or equal to +1 were considered as suppressor hits. All mutants with an average $Z$-score, between three replicates, less than or equal to -1 were considered as synthetic sick hits. Data are provided in Supplementary Data 3. The R script is provided in the Supplementary Software 1.

Drug sensitivity assays. Cells were grown to saturation in YPD or synthetic media containing raffinose and diluted in fresh medium at a cell concentration of $\sim 10^{7} \mathrm{cells} / \mathrm{ml}$. Ten-fold serial dilutions (unless specified otherwise) of the fresh cell suspension were then spotted on the appropriate agar plates with or without drug and incubated at $30^{\circ} \mathrm{C}$ unless specified otherwise. For acetaldehyde plate preparation, the drug was spread on $25 \mathrm{ml}$ YPD plates immediately before spotting the cells. Plates were photographed after 3-4 days or 7 days of incubation for YPD and synthetic media plates respectively.

Microscopy. For YOX1 mRNA imaging, cells were grown overnight in synthetic medium lacking methionine in order to induce expression of the MS2 coat protein and then diluted in low-fluorescence medium containing methionine $4-6 \mathrm{~h}$ prior to imaging. Where indicated, cells were treated with $\mathrm{HU}$ for $2 \mathrm{~h}$. Cells were imaged on a Leica DMI6000 confocal microscope.

For Yox1-GFP protein imaging, cells were first grown in synthetic complete medium overnight in a 96-well plate and diluted the next day for a second overnight culture in low-fluorescence synthetic complete medium. Cells were finally diluted to an $\mathrm{OD}_{600}$ of 0.05 in fresh low-fluorescence synthetic complete medium in a 384-well imaging plate $2 \mathrm{~h}$ prior to imaging and incubated at $30^{\circ} \mathrm{C}$ Cells were imaged on an Opera confocal fluorescence microscope (PerkinElmer) in absence of drug and 2 and $4 \mathrm{~h}$ after addition of $\mathrm{HU}$ at a final concentration of $200 \mathrm{mM}$.

For Rnr3-GFP protein imaging, cells in exponential phase grown in complete low-fluorescence medium for $17-19 \mathrm{~h}$ were imaged on a Leica DMI6000 confocal microscope.

Image analysis. For Yox1-GFP fluorescence quantification, image files from the Opera were analyzed using CellProfiler v2.1.1 ${ }^{68}$. Cell objects or nucleus objects were segmented using the Rpl39-tdTomato and Hta2-mCherry signals, respectively. GFP, tdTomato, and mCherry intensities were then measured in the segmented object. Median intensity measurements generated with CellProfiler were then exported for further analysis in R using customized pipelines. Yox1-GFP median intensities in the segmented cells or nuclei were normalized to Rpl39tdTomato or Hta2-mCherry median intensities, respectively. CellProfiler pipelines and the R script are included in the Supplementary Material.

For Rnr3-GFP fluorescence quantification, image files from the Volocity software v6.3 (PerkinElmer) were exported to tif format and analyzed using CellProfiler v2.1.1 ${ }^{68}$. Cell objects were segmented using the Rpl39-tdTomato signal. GFP intensity was then measured in the segmented object. Median intensity measurements generated with CellProfiler were then exported for further analysis in R using customized pipelines. CellProfiler pipelines and the R script are included in the Supplementary Software 1.

Protein extraction and immunoblot analysis. Protein extraction and immunoblotting was carried out as previously described ${ }^{69}$. Briefly, exponentially growing cells in YPD were collected before and after addition of HU at a final concentration of $200 \mathrm{mM}$. Cells were then fixed with trichloroacetic acid ( $10 \%$ final) for $15 \mathrm{~min}$ and whole-cells extracts prepared. Proteins were separated on a SDS-PAGE gel and immunoblots were performed using the FLAG-M2 antibody (Sigma, \#F3165) diluted 1:5000 or PGK1 antibody (Novex, \#4592250) diluted 1:1,000,000. An uncropped scan of the membrane is included in Supplementary Fig. 5.

RNA extraction and sequencing for cells overexpressing YOX1. Cells were grown to mid-logarithmic phase in synthetic complete medium lacking uracil and containing $2 \%$ raffinose, sampled, and then YOX1 overexpression was induced by the addition of galactose to $2 \%$ final, cultures were treated with $200 \mathrm{mM} \mathrm{HU}$, and sampled after 2 and $4 \mathrm{~h}$. Approximately $20 \mathrm{OD}_{600}$ of cell culture was used for RNA extraction. RNA extractions, RNA-seq, and data analysis was performed as described above. Mean FPKM values, $\log 2$ ratios, and $P$-values are provided in Supplementary Data 4. Each RNA-sequencing experiment included two independent biological replicates. Correlation between replicates was always $>0.92$. Raw sequencing data are accessible on GEO Omnibus with the accession number GSE83515.

qRT-PCR. RNA extraction was performed as described for the RNA-Seq experiments. Reverse transcription was performed using the Superscript II Reverse Transcriptase (Thermo Fischer Scientific) on $\sim 100$ ng of total RNA with the following gene specific reverse primers at a final concentration of $0.25 \mu \mathrm{M}$ : RLO62 (5'-ACGGGAGTCAACGTCTTCTT-3) for YOX1 mRNA, RLO198 (5'-TCTTGGC GCCTTCTTTCTTA-3) for ALD6 mRNA, and RLO25 (5'-CTTCTTAGCCTTAGCCATTT-3) for RPL39 mRNA. qPCR was then performed on $2 \mu \mathrm{l}$ of cDNA in a PCR reaction volume of $25 \mu \mathrm{l}$ using the SYBR Green PCR Master Mix (Thermo Fischer Scientific) and with the following primer pairs at a final concentration of $0.1 \mu \mathrm{M}$ : RLO61 (5'-CAAGGCGGACATACTTCGTG-3) and RLO62 for YOX1 mRNA, RLO197 (5'-ACGACGAACTATTGGCTGCT-3) and RLO198 for ALD6 mRNA, RLO24 (5'-GTGGTCCAGCGTGGTTTATG-3) and RLO25 for RPL39 mRNA. Differential expression was then assessed using the standard $\triangle \mathrm{Ct}$ method using RPL39 mRNA as internal standard. RPL39 standard was chosen as it was not differentially expressed in $l s m 1 \Delta$ cells or upon HU exposure in our RNASeq experiments. 
Growth kinetics. Strains were first grown to saturation in liquid medium in a 96-well plate and then diluted to an $\mathrm{OD}_{600}$ of 0.5 in fresh medium. An aliquot of $10 \mu \mathrm{l}$ of this cell suspension was then used to inoculate $90 \mu \mathrm{l}$ of fresh medium containing $\mathrm{HU}$ in a 96 -well plate. Cell growth was monitored by measuring $\mathrm{OD}_{600}$ every $15 \mathrm{~min}$ for $96 \mathrm{~h}$ using a Sunrise (Tecan) 96 -well plate OD reader. $\mathrm{OD}_{600}$ data were retrieved and further analyzed in $\mathrm{R}$ using a customized pipeline. The $\mathrm{R}$ pipeline is provided in the Supplementary Software 1. Briefly, cell growth was assessed by measuring the area under the growth curve $\left(\mathrm{OD}_{600}\right.$ as a function of time) and growth data were expressed as a percentage of growth for a given mutant compared to the control strain grown under the same conditions and during the same experiment. The experiment was repeated at least three times and included at least two independent biological replicates every time.

HU sensitivity of Yox1 target double mutants. Double mutant construction and growth monitoring: Deletion mutants, available in the yeast deletion collection ${ }^{62}$ and corresponding to the identified Yox1 targets (ald6 $\Delta, i c s 2 \Delta, m m r 1 \Delta$, pry $3 \Delta$, $\operatorname{rad} 54 \Delta$, uth $1 \Delta, y m l 131 W \Delta$ ) or the his $3 \Delta$ control strain were crossed with the following SGA query strains: ald6 $\Delta$ (RLKY105), mmr1 $\Delta$ (RLKY108), pry $3 \Delta$

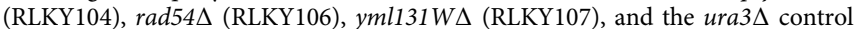
strain (RLKY109). After diploid selection and sporulation, haploid double mutants were selected using the SGA protocol ${ }^{28}$. uth $1 \Delta$ and $i c s 2 \Delta$ query mutants were excluded from the analysis as they did not display appropriate genetic linkage. Haploid double mutants were first grown to saturation in SGA double selection liquid medium and then diluted 1:100 in $90 \mu \mathrm{l}$ of YPD or YPD containing HU at a concentration of $25 \mathrm{mM}$ in a 96-well plate. Cell growth was then monitored by measuring $\mathrm{OD}_{600}$ every 15 min for $24 \mathrm{~h}$ using a Genios (Tecan) 96-well plate OD reader. The experiment was performed 5 times.

Genetic interaction assessment: For each growth condition (YPD or YPD+HU) we calculated the fitness $(\varepsilon)$ of each strain by measuring the area under the growth curve (OD600 as a function of time). The $\varepsilon$ value for each mutant was then normalized to the $\varepsilon$ value obtained for the control strain (his $3 \Delta$ ura $3 \Delta$ ). Negative genetic interactions were then assessed by comparing the observed fitness for each double mutant to the expected fitness calculated by multiplying the observed fitness for each of the two individual single mutants. Statistical significance was assessed by comparing expected and observed fitness values using the Student's $t$-test. In order to assess whether genetic interactions were exacerbated by HU treatment, we tested whether the observed fitness (relative to WT) of each double mutant displayed a statistically supported lower fitness in HU than in the untreated condition. Fitness data and $P$-values are provided in Supplementary Data 5.

Acetaldehyde-hydroxyurea drug interaction. Experimental design: Drug interactions were tested as previously described, with minor modifications ${ }^{70}$. Each drug was prepared at a $2 \times$ concentration in YPD (acetaldehyde $1 \%$ and HU $200 \mathrm{mM}$ ). An aliquot of $50 \mu \mathrm{l}$ of 2:3 serially diluted $\mathrm{HU}$ or $4: 5$ serially diluted acetaldehyde were then mixed together in 96-well plate in order to create a $6 \times 6$ array allowing testing of all drug combinations possible. In order to test the effect of each drug alone, serially diluted drugs were mixed with $50 \mu$ l of YPD. A no drug control was also included in the array design. An aliquot of $10 \mu \mathrm{l}$ of a saturated yeast BY4741 culture, diluted to an $\mathrm{OD}_{600}$ of 0.8 , was added to each well, and growth was monitored by measuring $\mathrm{OD}_{600}$ every $15 \mathrm{~min}$ for $24 \mathrm{~h}$ using a Genios (Tecan) 96-well plate OD reader. The experiment was performed three times.

Data analysis: For each condition (drug alone, drug combination, no drug), the fitness $(\varepsilon)$ of yeast cells was evaluated by measuring the area under the growth curve (OD600 as a function of time). The $\varepsilon$ value for each condition was then normalized to the $\varepsilon$ value obtained for the yeast cells grown in absence of drug. The interaction between acetaldehyde and HU was then evaluated by substracting the expected fitness (calculated by multiplying the normalized fitness values obtained for each drug alone at a given concentration) by the observed fitness value in presence of the combination of the two drugs. In this setting, negative values indicate synergy between acetaldehyde and $\mathrm{HU}$.

Cell-cycle analysis. Saturated cultures containing WT or $l s m 1 \Delta$ (JTY16) were diluted in fresh YPD or YPD containing HU at a final concentration of $200 \mathrm{mM}$. Samples for flow-cytometry were taken every hour during $4 \mathrm{~h}$. When cell synchronization was required (ald $6 \Delta$ experiment), G1 was induced for $3 \mathrm{~h}$ on exponentially growing cells using $\alpha$-factor at a concentration of $2.5 \mu \mathrm{g} / \mathrm{ml}$. G1 release was achieved by treating the culture with Pronase at a concentration of $100 \mu \mathrm{g} / \mathrm{ml}$. Sample preparation for flow-cytometry analysis was performed as previously described $^{12}$. Flow cytometric data were acquired on a FACSCanto II (BD Biosciences) and analyzed using the FlowJo software.

Data availability. All data generated or analyzed during this study are included in this published article (and its supplementary information files) except for the raw RNA-sequencing files that are available in the GEO Omnibus database with the accession number GSE83515 (https://www.ncbi.nlm.nih.gov/geo/). All the scripts generated to analyze the data during this study are included in the supplementary information files of this article.
Received: 8 December 2016 Accepted: 12 July 2017

Published online: 15 September 2017

\section{References}

1. Zeman, M. K. \& Cimprich, K. A. Causes and consequences of replication stress. Nat. Cell. Biol. 16, 2-9 (2014).

2. Truong, L. N. \& Wu, X. Prevention of DNA re-replication in eukaryotic cells. J. Mol. Cell Biol. 3, 13-22 (2011).

3. Segurado, M. \& Tercero, J. A. The S-phase checkpoint: targeting the replication fork. Biol. Cell 101, 617-627 (2009).

4. Chang, M., Bellaoui, M., Boone, C. \& Brown, G. W. A genome-wide screen for methyl methanesulfonate-sensitive mutants reveals genes required for $\mathrm{S}$ phase progression in the presence of DNA damage. Proc. Natl Acad. Sci. USA 99 16934-16939 (2002).

5. Gasch, A. P. et al. Genomic expression responses to DNA-damaging agents and the regulatory role of the yeast ATR homolog Meclp. Mol. Biol. Cell. 12, 2987-3003 (2001).

6. Dubacq, C. et al. Role of the iron mobilization and oxidative stress regulons in the genomic response of yeast to hydroxyurea. Mol. Genet. Genomic 275, 114-124 (2006).

7. Jaehnig, E. J., Kuo, D., Hombauer, H., Ideker, T. G. \& Kolodner, R. D. Checkpoint kinases regulate a global network of transcription factors in response to DNA damage. Cell Rep. 4, 174-188 (2013).

8. Tkach, J. M. et al. Dissecting DNA damage response pathways by analysing protein localization and abundance changes during DNA replication stress. Nat. Cell. Biol. 14, 966-976 (2012)

9. Chong, Y. T. et al. Yeast proteome dynamics from single cell imaging and automated analysis. Cell 161, 1413-1424 (2015).

10. Smolka, M. B., Albuquerque, C. P., Chen, S.-h \& Zhou, H. Proteome-wide identification of in vivo targets of DNA damage checkpoint kinases. Proc. Natl Acad. Sci. USA 104, 10364-10369 (2007).

11. Dénervaud, N. et al. A chemostat array enables the spatio-temporal analysis of the yeast proteome. Proc. Natl Acad. Sci. USA 110, 15842-15847 (2013).

12. Mazumder, A., Pesudo, L. Q., McRee, S., Bathe, M. \& Samson, L. D. Genome-wide single-cell-level screen for protein abundance and localization changes in response to DNA damage in S. cerevisiae. Nucleic Acids Res. 41, 9310-9324 (2013).

13. Jain, S. \& Parker, R. The discovery and analysis of P bodies. Adv. Exp. Med. Biol. 768, 23-43 (2013).

14. Teixeira, D., Sheth, U., Valencia-Sanchez, M.a., Brengues, M. \& Parker, R. Processing bodies require RNA for assembly and contain nontranslating mRNAs. RNA 11, 371-382 (2005).

15. Hendry, J. A., Tan, G., Ou, J., Boone, C. \& Brown, G. W. Leveraging DNA damage response signaling to identify yeast genes controlling genome stability. G3 5, 997-1006 (2015)

16. Boeck, R., Lapeyre, B., Brown, C. E. \& Sachs, A. B. Capped mRNA degradation intermediates accumulate in the yeast spb8-2 mutant. Mol. Cell Biol. 18, 5062-5072 (1998).

17. Bouveret, E., Rigaut, G., Shevchenko, A., Wilm, M. \& Séraphin, B. A Sm-like protein complex that participates in mRNA degradation. EMBO J. 19, 1661-1671 (2000).

18. Teixeira, D. \& Parker, R. Analysis of P-body assembly in Saccharomyces cerevisiae. Mol. Biol. Cell 18, 2274-2287 (2007).

19. Trapnell, C. et al. Differential gene and transcript expression analysis of RNA-seq experiments with TopHat and Cufflinks. Nat. Protoc. 7, 562-578 (2012).

20. He, W. \& Parker, R. The yeast cytoplasmic LsmI/Patlp complex protects mRNA 3' termini from partial degradation. Genetics 158, 1445-1455 (2001).

21. Sun, M. et al. Global analysis of Eukaryotic mRNA degradation reveals Xrn1-dependent buffering of transcript levels. Mol. Cell 52, 52-62 (2013).

22. Shalem, O. et al. Transient transcriptional responses to stress are generated by opposing effects of mRNA production and degradation. Mol. Syst. Biol. 4, 223 (2008).

23. Leng, N. et al. EBSeq: an empirical Bayes hierarchical model for inference in RNA-seq experiments. Bioinformatics 29, 1035-1043 (2013).

24. Robinson, M. D., McCarthy, D. J. \& Smyth, G. K. edgeR: a bioconductor package for differential expression analysis of digital gene expression data. Bioinformatics 26, 139-140 (2010).

25. Stark, C. et al. BioGRID: a general repository for interaction datasets. Nucleic Acids Res. 34, D535-D539 (2006).

26. Gasch, A. P. et al. Genomic expression programs in the response of yeast cells to environmental changes. Mol. Biol. Cell 11, 4241-4257 (2000).

27. Costanzo, M. et al. A global genetic interaction network maps a wiring diagram of cellular function. Science 353, aaf1420 (2016).

28. Tong, A. \& Boone, C. High-throughput strain construction and systematic synthetic lethal screening in Saccharomyces cerevisiae. Methods Mol. Biol. 36, 1-19 (2005). 
29. Tsuno, A., Miyoshi, K., Tsujii, R., Miyakawa, T. \& Mizuta, K. RRS1, a conserved essential gene, encodes a novel regulatory protein required for ribosome biogenesis in Saccharomyces cerevisiae. Mol. Cell Biol. 20, 2066-2074 (2000).

30. Fleischer, T. C., Weaver, C. M., McAfee, K. J., Jennings, J. L. \& Link, A. J. Systematic identification and functional screens of uncharacterized proteins associated with eukaryotic ribosomal complexes. Genes Dev. 20, 1294-1307 (2006).

31. Ubersax, J. A. et al. Targets of the cyclin-dependent kinase Cdk1. Nature 425, 859-864 (2003).

32. Herrero, A. B. \& Moreno, S. Lsm1 promotes genomic stability by controlling histone mRNA decay. EMBO J. 30, 2008-2018 (2011).

33. Pramila, T., Miles, S., Guhathakurta, D., Jemiolo, D. \& Breeden, L. L. Conserved homeodomain proteins interact with MADS box protein $\mathrm{Mcm} 1$ to restrict ECB-dependent transcription to the M/G1 phase of the cell cycle. Genes Dev. 16, 3034-3045 (2002).

34. Gomez-Escoda, B. et al. Yox1 links MBF-dependent transcription to completion of DNA synthesis. EMBO Rep. 12, 84-89 (2011).

35. Braun, K. A. \& Breeden, L. L. Nascent transcription of MCM2-7 is important for nuclear localization of the minichromosome maintenance complex in G1. Mol. Biol. Cell 18, 1447-1456 (2007).

36. Schmidt, M., Akasaka, K., Messerly, J. T. \& Boyer, M. P. Role of Hog1, Tps1 and Sod1 in boric acid tolerance of Saccharomyces cerevisiae. Microbiology 158, 2667-2678 (2012).

37. Mayes, A. E., Verdone, L., Legrain, P. \& Beggs, J. D. Characterization of Sm-like proteins in yeast and their association with U6 snRNA. EMBO J. 18, 4321-4331 (1999).

38. Haim, L., Zipor, G., Aronov, S. \& Gerst, J. E. A genomic integration method to visualize localization of endogenous mRNAs in living yeast. Nat. Methods 4 , 409-412 (2007)

39. Garcia, J. F. \& Parker, R. MS2 coat proteins bound to yeast mRNAs block $5^{\prime}$ to 3 ' degradation and trap mRNA decay products: implications for the localization of mRNAs by MS2-MCP system. RNA 21, 1393-1395 (2015).

40. Garcia, J. F. \& Parker, R. Ubiquitous accumulation of 3' mRNA decay fragments in Saccharomyces cerevisiae mRNAs with chromosomally integrated MS2 arrays. RNA 22, 657-659 (2016).

41. Sheth, U. \& Parker, R. Decapping and decay of messenger RNA occur in cytoplasmic processing bodies. Science 300, 805-808 (2003).

42. Lavut, A. \& Raveh, D. Sequestration of highly expressed mRNAs in cytoplasmic granules, P-bodies, and stress granules enhances cell viability. PLoS Genet. 8, e1002527-e1002527 (2012).

43. Teixeira, M. C. et al. The YEASTRACT database: an upgraded information system for the analysis of gene and genomic transcription regulation in Saccharomyces cerevisiae. Nucleic Acids Res. 42, D161-D166 (2014).

44. de Boer, C. G. \& Hughes, T. R. YeTFaSCo: a database of evaluated yeast transcription factor sequence specificities. Nucleic Acids Res. 40, D169-D179 (2012).

45. Luo, Z., Walkey, C. J., Madilao, L. L., Measday, V. \& Van Vuuren, H. J. Functional improvement of Saccharomyces cerevisiae to reduce volatile acidity in wine. FEMS Yeast Res. 13, 485-494 (2013).

46. Kim, H. \& D'Andrea, A. D. Regulation of DNA cross-link repair by the Fanconi anemia/BRCA pathway. Genes Dev. 26, 1393-1408 (2012).

47. Eulalio, A., Behm-Ansmant, I., Schweizer, D. \& Izaurralde, E. P-body formation is a consequence, not the cause, of RNA-mediated gene silencing. Mol. Cell Biol. 27, 3970-3981 (2007)

48. Decker, C. J., Teixeira, D. \& Parker, R. Edc3p and a glutamine/asparagine-rich domain of Lsm4p function in processing body assembly in Saccharomyces cerevisiae. J. Cell Biol. 179, 437-449 (2007).

49. Haimovich, G. et al. Gene expression is circular: factors for mRNA degradation also foster mRNA synthesis. Cell 153, 1000-1011 (2013).

50. Decker, C. J. \& Parker, R. P-bodies and stress granules: possible roles in the control of translation and mRNA degradation. Cold Spring Harb. Perspect. Biol. 4, a012286 (2012).

51. Nyholm, S., Thelander, L. \& Graslund, A. Reduction and loss of the iron center in the reaction of the small subunit of mouse ribonucleotide reductase with hydroxyurea. Biochemistry 32, 11569-11574 (1993).

52. Chitambar, C. R. \& Wereley, J. P. Effect of hydroxyurea on cellular iron metabolism in human leukemic CCRF-CEM cells: changes in iron uptake and the regulation of transferrin receptor and ferritin gene expression following inhibition of DNA synthesis. Cancer Res. 55, 4361-4366 (1995).

53. Zhang, A. et al. The human long non-coding RNA-RoR is a p53 repressor in response to DNA damage. Cell Res. 23, 340-350 (2013).

54. Lamaa, A. et al. A novel cytoprotective function for the DNA repair protein $\mathrm{Ku}$ in regulating p53 mRNA translation and function. EMBO Rep. 17, 508-518 (2016).

55. Powley, I. R. et al. Translational reprogramming following UVB irradiation is mediated by DNA-PKcs and allows selective recruitment to the polysomes of mRNAs encoding DNA repair enzymes. Genes Dev. 23, 1207-1220 (2009).

56. Braunstein, S., Badura, M. L., Xi, Q., Formenti, S. C. \& Schneider, R. J. Regulation of protein synthesis by ionizing radiation. Mol. Cell Biol. 29, 5645-5656 (2009)
57. Loveless, T. B. et al. DNA damage regulates translation through $\beta$-TRCP targeting of CReP. PLoS Genet. 11, e1005292-e1005292 (2015).

58. Bai, G., Smolka, M. B. \& Schimenti, J. C. Chronic DNA replication stress reduces replicative lifespan of cells by TRP53-dependent, microRNA-assisted MCM2-7 downregulation. PLoS Genet. 12, e1005787-e1005787 (2016).

59. Birrell, G. W. et al. Transcriptional response of Saccharomyces cerevisiae to DNA-damaging agents does not identify the genes that protect against these agents. Proc. Natl Acad. Sci. USA 99, 8778-8783 (2002).

60. Aranda, A. \& del Olmo, M.l. Response to acetaldehyde stress in the yeast Saccharomyces cerevisiae involves a strain-dependent regulation of several $A L D$ genes and is mediated by the general stress response pathway. Yeast 20 747-759 (2003).

61. Michl, J., Zimmer, J., Buffa, F. M., McDermott, U. \& Tarsounas, M. FANCD2 limits replication stress and genome instability in cells lacking BRCA2. Nat. Struct. Mol. Biol. 23, 755-757 (2016).

62. Giaever, G. et al. Functional profiling of the Saccharomyces cerevisiae genome Nature. 418, 387-391 (2002).

63. Brachmann, C. B. et al. Designer deletion strains derived from Saccharomyces cerevisiae S288C: a useful set of strains and plasmids for PCR-mediated gene disruption and other applications. Yeast 14, 115-132 (1998).

64. Kim, D. et al. TopHat2: accurate alignment of transcriptomes in the presence of insertions, deletions and gene fusions. Genome Biol. 14, R36-R36 (2013).

65. Supek, F., Bošnjak, M., Škunca, N. \& Šmuc, T. REVIGO summarizes and visualizes long lists of gene ontology terms. PLOS ONE 6, e21800-e21800 (2011).

66. Shannon, P. et al. Cytoscape: a software environment for integrated models of biomolecular interaction networks. Genome Res. 13, 2498-2504 (2003).

67. Wagih, O. et al. SGAtools: one-stop analysis and visualization of array-based genetic interaction screens. Nucleic Acids Res. 41, W591-W596 (2013).

68. Kamentsky, L. et al. Improved structure, function and compatibility for CellProfiler: modular high-throughput image analysis software. Bioinformatics 27, 1179-1180 (2011).

69. Balint, A. et al. Assembly of Slx 4 signaling complexes behind DNA replication forks. EMBO J. 34, 2182-2197 (2015).

70. Torres, N. P., Lee, A. Y., Giaever, G., Nislow, C. \& Brown, G. W. A highthroughput yeast assay identifies synergistic drug combinations. Assay. Drug. Dev. Technol. 11, 299-307 (2013).

\section{Acknowledgements}

We thank Guihong Tan and Charlie Boone for generously providing the temperature sensitive yeast strain array. We thank Dax Torti and the Donnelly Sequencing Centre for RNA-Seq library preparation and sequencing. We are very grateful to all the members of the Brown lab for helpful discussions and to Craig Smibert for his critical reading of the manuscript. This work was supported by Canadian Institutes of Health Research grant MOP-137043 and by a Cancer Research Society grant (to G.W.B.).

\section{Author contributions}

Conceptualization: R.L.-K. and G.W.B.; methodology: R.L.-K.; formal analysis: R.L.-K.; writing and original draft: R.L.-K.; writing, review, and editing: R.L.-K. and G.W.B.

\section{Additional information}

Supplementary Information accompanies this paper at doi:10.1038/s41467-017-00632-2.

Competing interests: The authors declare no competing financial interests.

Reprints and permission information is available online at http://npg.nature.com/ reprintsandpermissions/

Publisher's note: Springer Nature remains neutral with regard to jurisdictional claims in published maps and institutional affiliations.

\footnotetext{
Open Access This article is licensed under a Creative Commons Attribution 4.0 International License, which permits use, sharing, adaptation, distribution and reproduction in any medium or format, as long as you give appropriate credit to the original author(s) and the source, provide a link to the Creative Commons license, and indicate if changes were made. The images or other third party material in this article are included in the article's Creative Commons license, unless indicated otherwise in a credit line to the material. If material is not included in the article's Creative Commons license and your intended use is not permitted by statutory regulation or exceeds the permitted use, you will need to obtain permission directly from the copyright holder. To view a copy of this license, visit http://creativecommons.org/ licenses/by/4.0/.
}

(C) The Author(s) 2017 